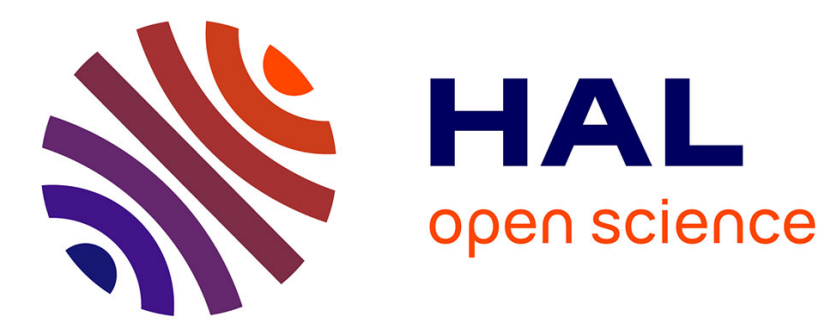

\title{
Finite Element solution of the fiber/matrix interface crack problem: convergence properties and mode mixity of the Virtual Crack Closure Technique
}

Luca Di Stasio, Zoubir Ayadi

\section{- To cite this version:}

Luca Di Stasio, Zoubir Ayadi. Finite Element solution of the fiber/matrix interface crack problem: convergence properties and mode mixity of the Virtual Crack Closure Technique. 2019. hal-02187502

\author{
HAL Id: hal-02187502 \\ https://hal.science/hal-02187502
}

Preprint submitted on 17 Jul 2019

HAL is a multi-disciplinary open access archive for the deposit and dissemination of scientific research documents, whether they are published or not. The documents may come from teaching and research institutions in France or abroad, or from public or private research centers.
L'archive ouverte pluridisciplinaire HAL, est destinée au dépôt et à la diffusion de documents scientifiques de niveau recherche, publiés ou non, émanant des établissements d'enseignement et de recherche français ou étrangers, des laboratoires publics ou privés.

\section{(ㅇ)(1) $\$$}

Distributed under a Creative Commons Attribution - NonCommercial - NoDerivatives| 4.0 


\title{
Finite Element solution of the fiber/matrix interface crack problem: convergence properties and mode mixity of the Virtual Crack Closure Technique
}

\author{
Luca Di Stasio ${ }^{\mathrm{a}, \mathrm{b}}$, Zoubir Ayadi $^{\mathrm{b}}$ \\ ${ }^{a}$ Luleå University of Technology, University Campus, SE-97187 Luleå, Sweden \\ ${ }^{b}$ Université de Lorraine, EEIGM, IJL, 6 Rue Bastien Lepage, F-54010 Nancy, France
}

\begin{abstract}
The bi-material interface arc crack has been the focus of interest in the composite community, where it is usually referred to as the fiber-matrix interface crack. In this work, we investigate the convergence properties of the Virtual Crack Closure Technique (VCCT) when applied to the evaluation of the Mode I, Mode II and total Energy Release Rate of the fiber-matrix interface crack in the context of the Finite Element Method (FEM). We first propose a synthetic vectorial formulation of the VCCT. Thanks to this formulation, we study the convergence properties of the method, both analytically and numerically. It is found that Mode 1 and Mode II Energy Release Rate (ERR) possess a logarithmic dependency with respect to the size of the elements in the crack tip neighborhood, while the total ERR is independent of element size.
\end{abstract}

Keywords: Fiber/matrix interface crack, Bi-material interface arc crack, Linear Elastic Fracture Mechanics (LEFM), Virtual Crack Closure Technique (VCCT), Mode separation, Convergence

\section{Introduction}

Bi-material interfaces represent the basic load transfer mechanism at the heart of Fiber Reinforced Polymer Composite (FRPC) materials. They are present at the macroscale, in the form of adhesive joints; at the mesoscale, as 5 interfaces between layers with different orientations; at the microscale, as fiber- 
matrix interfaces. Bi-material interfaces have for long attracted the attention of researchers in Fracture Mechanics [1, 2], due to their hidden complexity.

The problem was first addressed in the 1950's by Williams [3], who derived through a linear elastic asymptotic analysis the stress distribution around an open crack (i.e. with crack faces nowhere in contact for any size of the crack) between two infinite half-planes of dissimilar materials. He found the existence of a strong oscillatory behavior in the stress singularity at the crack tip of the form

$$
r^{-\frac{1}{2}} \sin (\varepsilon \log r) \quad \text { with } \quad \varepsilon=\frac{1}{2 \pi} \log \left(\frac{1-\beta}{1+\beta}\right),
$$

in both Mode I and Mode II. In Eq. 1] $\beta$ is one of the two parameters introduced by Dundurs 4 to characterize bi-material interfaces:

$$
\beta=\frac{\mu_{2}\left(\kappa_{1}-1\right)-\mu_{1}\left(\kappa_{2}-1\right)}{\mu_{2}\left(\kappa_{1}+1\right)+\mu_{1}\left(\kappa_{2}+1\right)}
$$

where $\kappa=3-4 \nu$ in plane strain and $\kappa=\frac{3-4 \nu}{1+\nu}$ in plane stress, $\mu$ is the shear modulus, $\nu$ Poisson's coefficient, and indexes 1,2 refer to the two bulk materials joined at the interface. Defining $a$ as the length of the crack, it was found that the size of the oscillatory region is in the order of $10^{-6} a$ [5]. Given the oscillatory behaviour of the crack tip singularity of Eq. 1, the definition of Stress Intensity Factor (SIF) $\lim _{r \rightarrow 0} \sqrt{2 \pi r} \sigma$ diverges and ceases to be valid [1]. It implies that the Mode mixity problem at the crack tip is ill-posed.

It was furthermore observed, always in the context of Linear Elastic Fracture Mechanics (LEFM), that an interpenetration zone exists close to the crack tip [6, $\left.{ }_{25} 7\right]$ with a length in the order of $10^{-4} a[6]$. Following conclusions firstly proposed in [7, the presence of a contact zone in the crack tip neighborhood, of a length to be determined from the solution of the elastic problem, was introduced in [8] and shown to provide a physically consistent solution to the straight bi-material interface crack problem.

30 The curved bi-material interface crack, more often refered to as the fiber-matrix interface crack (or debond) due to its relevance in FRPCs, was first treated by 
England [9] and by Perlman and Sih [10], who provided the analytical solution of stress and displacement fields for a circular inclusion with respectively a single debond and an arbitrary number of debonds. Building on their work, Toya [11] particularized the solution and provided the expression of the Energy Release Rate (ERR) at the crack tip. The same problems exposed previously for the open straight bi-material crack were shown to exist also for the open fiber-matrix interface crack: the presence of strong oscillations in the crack tip singularity and onset of crack face interpenetration at a critical flaw size ${ }^{1}$

40 In order to treat cases more complex than the single partially debonded fiber in an infinite matrix of [9, 10, 11, numerical studies followed. In the 1990's, París and collaborators [12 developed a Boundary Element Method (BEM) with the use of discontinuous singular elements at the crack tip and the Virtual Crack Closure Integral (VCCI) 13. for the evaluation of the Energy Release ${ }_{45}$ Rate (ERR). They validated their results [12] with respect to Toya's analytical solution [11 and analyzed the effect of BEM interface discretization on the stress field in the neighborhood of the crack tip [14]. Following Comninou's work on the straight crack [8], they furthermore recognized the importance of contact to retrieve a physical solution avoiding interpenetration [12] and studied the effect of the contact zone on debond ERR [15]. Their algorithm was then applied to investigate the fiber-matrix interface crack under different geometrical configurations and mechanical loadings $[16,17,18,19,20,21,22$.

Recently the Finite Element Method (FEM) was also applied to the solution of the fiber-matrix interface crack problem [23, 24, 25], in conjunction with the Virtual Crack Closure Technique (VCCT) [26, 27] for the evaluation of the ERR at the crack tip. In [23, the authors validated their model with respect to the BEM results of [12, but no analysis of the effect of the discretization in the crack tip neighborhood comparable to [14] was proposed. Thanks to the interest in evaluating the ERR of interlaminar delamination, different studies

\footnotetext{
${ }^{1}$ For the fiber-matrix interface crack, flaw size is measured in terms of the angle $\Delta \theta$ subtended by half of the arc-crack, i.e. $a=2 \Delta \theta$.
} 
exist in the literature on the effect of mesh discretization on Mode I and Mode II ERR of the bi-material interface crack when evaluated with the VCCT in the context of the FEM [28, 29, 30. However, no comparable analysis can be found in the literature on mode separation and convergence analysis of the VCCT when applied to the fiber-matrix interface crack (circular bi-material interface crack) problem in the context of a linear elastic FEM solution. In the present article, we first present the FEM formulation of the problem, together with the main geometrical characteristics, material properties, boundary conditions and loading. We then propose a vectorial formulation of the VCCT and express Mode I and Mode II ERR in terms of FEM natural variables. With this tool, we derive an analytical estimate of the ERR convergence and compare it with numerical results.

\section{FEM formulation of the fiber-matrix interface crack problem}

In order to investigate the fiber-matrix interface crack problem, a 2-dimensional model of a single fiber inserted in a rectangular matrix element is considered (see

${ }_{75}$ Figure 1). Total element length and height are respectively $2 L$ and $L$, where $L$ is determined by the fiber radius $R_{f}$ and the fiber volume fraction $V_{f}$ by

$$
L=\frac{R_{f}}{2} \sqrt{\frac{\pi}{V_{f}}} .
$$

The fiber radius $R_{f}$ is assumed to be equal to $1 \mu \mathrm{m}$. This choice is not dictated by physical considerations but for simplicity. It is thus useful to remark that, in a linear elastic solution as the one considered in the present work, the ERR is proportional to the geometrical dimensions of the model and, consequently, recalculation of the ERR for fibers of any size requires a simple multiplication.

As shown in Fig. 1, the debond is placed symmetrically with respect to the $x$ axis and its size is characterized by the angle $\Delta \theta$ (which makes the full debond size equal to $2 \Delta \theta$ and the full crack length equal to $R_{f} 2 \Delta \theta$ ). A region $\Delta \Phi$ of unknown size appears at the crack tip for large debond sizes (at least 


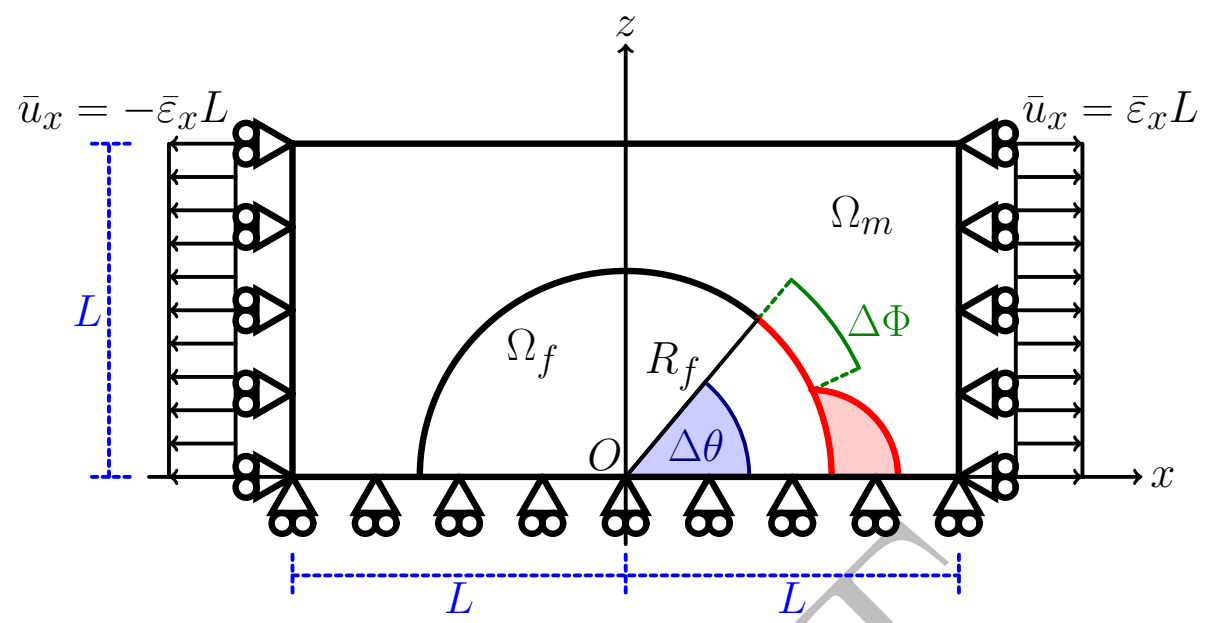

Figure 1: Schematic of the model with its main parameters.

$\geq 60^{\circ}-80^{\circ}$ ), in which the crack faces are in contact with each other and free to slide. Frictionless contact is thus considered between the two crack faces to allow free sliding and avoid interpenetration. Symmetry with respect to the $x$ axis is applied on the lower boundary while the upper surface is left free. Kinematic coupling on the $x$-displacement is applied along the left and right sides of the model in the form of a constant $x$-displacement $\pm \bar{\varepsilon}_{x} L$, which corresponds to transverse strain $\bar{\varepsilon}_{x}$ equal to $1 \%$ in the results here presented.

Table 1: Summary of the mechanical properties of fiber and matrix. E stands for Young's modulus, $\mu$ for shear modulus and $\nu$ for Poisson's ratio.

\begin{tabular}{cccc} 
Material & $E[G P a]$ & $\mu[G P a]$ & $\nu[-]$ \\
\hline Glass fiber & 70.0 & 29.2 & 0.2 \\
Epoxy & 3.5 & 1.25 & 0.4
\end{tabular}

The model problem is solved with the Finite Element Method (FEM) within the Abaqus environment, a commercial FEM software [31. The model is meshed with second order, 2D, plane strain triangular (CPE6) and rectangular (CPE8) elements. A regular mesh of rectangular elements with almost unitary aspect ratio is used at the crack tip. The angular size $\delta$ of an element in the crack 
tip neighborhood represents the main parameter of the numerical analysis. The crack faces are modeled as element-based surfaces and a small-sliding contact pair interaction with no friction is imposed between them. The Mode I, Mode II and total Energy Release Rates (ERRs) (respectively referred to as $G_{I}, G_{I I}$ and $\left.G_{T O T}\right)$ are evaluated using the VCCT [27], implemented in a in-house Python routine. A glass fiber-epoxy system is considered in the present work, and it is assumed that their response lies always in the linear elastic domain. The elastic properties of glass fiber and epoxy are reported in Table 1 .

\section{Vectorial formulation of the Virtual Crack Closure Technique (VCCT)}

In order to express the VCCT formulation of the ERR in terms of FEM variables, we need to introduce a few rotation matrices in order to represent the discretized representation (FE mesh) of a crack along a circular interface. The position of the crack tip is characterized by the angular size of the crack (see Sec. 2 and Fig. 1 for reference) and the rotation corresponding to the crack tip reference frame is represented by the matrix $\underline{\underline{R}}_{\Delta \theta}$ defined as

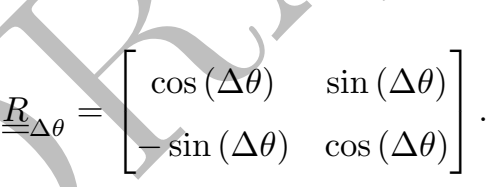

Nodes belonging to the elements sharing the crack tip are involved in the VCCT estimation of the ERR and it is assumed that, given a sufficiently fine discretization, they are aligned with the crack propagation direction defined at the crack tip. However, irrespectively of how small the elements in the crack tip neighborhood are, a misalignment always exists with respect to the assumed crack propagation direction (in the crack tip reference frame). This is measured by the matrices $\underline{\underline{P}}_{\delta}(p)$, defined as

$$
\underline{\underline{P}}_{\delta}(p)=\left[\begin{array}{cc}
\cos \left(\left(1+\frac{1-p}{m}\right) \delta\right) & \sin \left(\left(1+\frac{1-p}{m}\right) \delta\right) \\
-\sin \left(\left(1+\frac{1-p}{m}\right) \delta\right) & \cos \left(\left(1+\frac{1-p}{m}\right) \delta\right)
\end{array}\right]
$$


and $\underline{\underline{Q}}_{\delta}(q)$, equal to

$$
\underline{\underline{Q}}_{\delta}(q)=\left[\begin{array}{cc}
\cos \left(\frac{q-1}{m} \delta\right) & \sin \left(\frac{q-1}{m} \delta\right) \\
-\sin \left(\frac{q-1}{m} \delta\right) & \cos \left(\frac{q-1}{m} \delta\right)
\end{array}\right],
$$

respectively for the free and bonded nodes involved in the VCCT estimation.

In Eqs. 5 and 6 , $\delta$ is the angular size of an element in the crack tip neighborhood (see Sec. 2 and Fig. 1), $m$ is the order of the element shape functions and $p, q$ are indices referring to the nodes belonging respectively to free and bonded elements sharing the crack tip. Introducing the permutation matrix

$$
\underline{\underline{P}}_{\pi}=\left[\begin{array}{cc}
0 & 1 \\
-1 & 0
\end{array}\right]
$$

it is possible to express the derivatives of rotation matrices $\underline{\underline{R}}_{\Delta \theta}, \underline{\underline{P}}_{\delta}$ and $\underline{\underline{Q}} \delta$ with respect to their argument:

$$
\frac{\partial \underline{\underline{R}}_{\Delta \theta}}{\partial \Delta \theta}=\underline{\underline{D}} \cdot \underline{\underline{R}}_{\Delta \theta}, \quad \frac{\partial \underline{\underline{P}}_{\delta}}{\partial \delta}=\left(1+\frac{1-p}{m}\right) \underline{\underline{D}} \cdot \underline{\underline{P}}_{\delta}, \quad \frac{\partial \underline{\underline{Q}} \delta}{\partial \delta}=\frac{q-1}{m} \underline{\underline{D}} \cdot \underline{\underline{Q}} \delta .
$$

By means of Eqs. 5 and 6 . we can express the crack tip forces $\underline{F}_{x y}=\left[\begin{array}{c}F_{x} \\ F_{y}\end{array}\right]$ 130 and crack displacements $\underline{u}_{x y}=\left[\begin{array}{l}u_{x} \\ u_{y}\end{array}\right]$ in the crack tip reference frame (where the tangential direction $\theta$ correspond to the direction of crack propagation) while taking into account the misalignment to the finite discretization as

$$
\underline{F}_{r \theta}=\underline{\underline{Q}}_{\delta} \underline{\underline{R}}_{\Delta \theta} \underline{F}_{x y} \quad \underline{u}_{r \theta}=\underline{\underline{P}}_{\delta} \underline{\underline{R}}_{\Delta \theta} \underline{u}_{x y}
$$

where $\underline{F}_{r \theta}=\left[\begin{array}{c}F_{r} \\ F_{\theta}\end{array}\right]$ and $\underline{u}_{r \theta}=\left[\begin{array}{l}u_{r} \\ u_{\theta}\end{array}\right]$.

The crack tip forces can be expressed as a function of the crack opening 135 displacement as

$$
\underline{F}_{x y}=\underline{\underline{K}}_{x y} \underline{u}_{x y}+\underline{\widetilde{F}}_{x y}
$$


where $\underline{\underline{K}}_{x y}$ is in general a full matrix of the form $\underline{\underline{K}}_{x y}=\left[\begin{array}{c}K_{x x} K_{x y} \\ K_{y x} K_{y y}\end{array}\right]$ and $\underline{\underline{F}}_{x y}$ represents the effect of the rest of the FE solution through the remaining nodes of the elements attached to the crack tip. As such, the term $\underline{\widetilde{F}}_{x y}$ can be expressed as a linear combination of the solution vector $\underline{u}_{N}$ of nodal displacements of the form $\underline{\underline{K}}_{N} \underline{u}_{N}$. Equation 10 thus become

$$
\underline{F}_{x y}=\underline{\underline{K}}_{x y} \underline{u}_{x y}+\underline{\underline{\widetilde{K}}}_{N} \underline{u}_{N} .
$$

An exemplifying derivation of the relationships expressed in Equations 10 and 11 can be found in Appendix A. It is worthwhile to observe that another author [32] proposed a relationship of the form $\underline{F}_{x y}=\underline{\underline{K}}_{x y} \underline{u}_{x y}$. However, in [32], this relationship is assumed a priori and manipulated to propose a revised version of the VCCT, based on the assumption that the matrix $\underline{\underline{K}}_{x y}$ should be diagonal to provide physically-consistent fracture mode partitioning. On the other hand, in the present work we derive the relationships of Eqs. 10 and 11 from the formulation of the Finite Element Method. According to our derivation, it seems correct that the matrix $\frac{K}{x} x y$ should not in general be diagonal in order to take into account Poisson's effect. In fact, a positive crack opening displacement would cause a transyerse displacement in the neighborhood of the crack tip. Given that material properties are different on the two sides of a bi-material interface, a net shear would be applied to the crack tip which would correspond to a net contribution to the crack tip force related to crack shear displacement. The analytical derivations presented in Appendix A confirm these physical considerations.

Based upon the work of Raju [33], we introduce the matrix $\underline{\underline{T}}_{p q}$ to represent the weights needed in the VCCT to account for the use of singular elements. As already done previously, indices $p$ and $q$ refer to nodes placed respectively on 160 the free (crack face) and bonded side of the crack tip. Nodes are enumerated so that the crack tip has always index 1, i.e. the higher the index the further the node is from the crack tip. Matrix $\underline{\underline{T}}_{p q}$ has always a size of $d \times d$, where 
$d=2$ for a $2 D$ problem and $d=3$ for a $3 D$ problem. An element $\underline{\underline{T}}_{p q}(i, j)$ with $i, j=1, \ldots, d$ represents the weight to be assigned to the product of component $i$ of the displacement extracted at node $p$ with component $j$ of the force extracted at node $q$. The expression of $\underline{\underline{T}}_{p q}$ for quadrilateral elements with or without singularity is reported in Appendix B. Notice that, given $m$ is the order of the element shape functions, the element side has $m+1$ nodes and this represents the upper limit of indices $p$ and $q$.

170 By using matrix $\underline{\underline{T}}_{p q}$, it is possible to express the total ERR $G$ evaluated with the VCCT as

$$
G_{T O T}=\frac{1}{2 R_{f} \delta} \sum_{p=1}^{m+1} \sum_{q=1}^{m+1} \operatorname{Tr}\left(\underline{u}_{r \theta, p}^{T} \underline{T}_{p q}^{T} \underline{F}_{r \theta, q}\right) .
$$

Introducing the vector $\underline{G}=\left[\begin{array}{c}G_{I} \\ G_{I I}\end{array}\right]$ of fracture mode ERRs, Mode I and Mode II ERR evaluated with the VCCT can be expressed as

$$
\underline{G}=\frac{1}{2 R_{f} \delta} \sum_{p=1}^{m+1} \sum_{q=1}^{m+1} \operatorname{Diag}\left(\underline{F}_{r \theta, q} \underline{u}_{r \theta, p}^{T} \underline{\underline{T}}_{p q}^{T}\right),
$$

where $\operatorname{Diag}()$ is the function that extracts the main diagonal of the input 175 matrix as a column vector. Substituting Equations 9 and 11 in Equations 12 and 13, we can express the Mode I, Mode II and total Energy Release Rate as a function of the crack displacements and the FE solution (more details in Appendix A as

$$
\begin{aligned}
G_{T O T}= & \frac{1}{2 R_{f} \delta} \sum_{p=1}^{m+1} \sum_{q=1}^{m+1} \operatorname{Tr}\left(\underline{\underline{Q}}_{\delta} \underline{\underline{R}}_{\Delta \theta} \underline{\underline{K}}_{x y, q} \underline{\underline{u}}_{x y, q} \underline{\underline{u}}_{x y, p}^{T} \underline{\underline{R}}_{\Delta \theta}^{T} \underline{\underline{P}}_{\delta}^{T} \underline{\underline{T}}_{p q}^{T}\right)+ \\
& +\frac{1}{2 R_{f} \delta} \sum_{p=1}^{m+1} \sum_{q=1}^{m+1} \operatorname{Tr}\left(\underline{\underline{Q}}_{\delta} \underline{\underline{R}}_{\Delta \theta} \underline{\widetilde{F}}_{x y, q} \underline{\underline{u}}_{x y, p}^{T} \underline{\underline{R}}_{\Delta \theta}^{T} \underline{\underline{P}}_{\delta}^{T} \underline{\underline{T}}_{p q}^{T}\right)
\end{aligned}
$$


and

$$
\begin{aligned}
\underline{G}=\left[\begin{array}{c}
G_{I} \\
G_{I I}
\end{array}\right]= & \frac{1}{2 R_{f} \delta} \sum_{p=1}^{m+1} \sum_{q=1}^{m+1} \operatorname{Diag}\left(\underline{\underline{Q}}_{\delta} \underline{\underline{R}}_{\Delta \theta} \underline{\underline{K}}_{x y, q} \underline{u}_{x y, q} \underline{u}_{x y, p}^{T} \underline{\underline{R}}_{\Delta \theta}^{T} \underline{\underline{P}}_{\delta}^{T} \underline{\underline{T}}_{p q}^{T}\right)+ \\
& +\frac{1}{2 R_{f} \delta} \sum_{p=1}^{m+1} \sum_{q=1}^{m+1} \operatorname{Diag}\left(\underline{\underline{Q}}_{\delta} \underline{\underline{R}}_{\Delta \theta} \underline{\underline{\widetilde{K}}}_{N, q} \underline{u}_{N} \underline{\underline{u}}_{x y, p}^{T} \underline{\underline{R}}_{\Delta \theta}^{T} \underline{\underline{P}}_{\delta}^{T} \underline{\underline{T}}_{p q}^{T}\right)
\end{aligned}
$$

180

\section{Rotational invariance of $G_{T O T}$}

Recalling Equation 14 and observing that matrix $\underline{\underline{T}}_{p q}$ is always equal to the identity matrix pre-multiplied by a suitable real constant (see Eq. B.1 in Appendix B, the total Energy Release Rate can be rewritten as

$$
\begin{aligned}
G_{T O T} & =\frac{1}{2 R_{f} \delta} \sum_{p=1}^{m+1} \sum_{q=1}^{m+1} \operatorname{Tr}\left(\underline{\underline{Q}}_{\delta} \underline{\underline{R}}_{\Delta \theta}\left(\underline{\underline{K}}_{x y, q} \underline{\underline{u}}_{x y, q}+\underline{\underline{F}}_{x y, q}\right) \underline{u}_{x y, p}^{T} \underline{\underline{T}}_{p q}^{T} \underline{\underline{R}}_{\Delta \theta}^{T} \underline{\underline{P}}_{\delta}^{T}\right)= \\
& =\frac{1}{2 R_{f} \delta} \sum_{p=1}^{m+1} \sum_{q=1}^{m+1} \operatorname{Tr}\left(\underline{\underline{Q}} \delta \underline{\underline{R}}_{\Delta \theta} \underline{F}_{x y, q} \underline{\underline{u}}_{x y, p}^{T} \underline{\underline{T}}_{p q}^{T} \underline{\underline{R}}_{\Delta \theta}^{T} \underline{\underline{P}}_{\delta}^{T}\right),
\end{aligned}
$$

where $\underline{F}_{x y}$ and $\underline{u}_{x y}$ are the vectors of respectively the crack tip forces and crack displacements in the global $(x-y)$ reference frame. Given that $\underline{\underline{Q}} \delta, \underline{\underline{P}} \delta$ and $\underline{\underline{R}}_{\Delta \theta}$ all represent a linear transformation (a rigid rotation in particular), the invariance of the trace to linear transformations ensures that

$$
\begin{aligned}
G_{T O T} & =\frac{1}{2 R_{f} \delta} \sum_{p=1}^{m+1} \sum_{q=1}^{m+1} \operatorname{Tr}\left(\underline{\underline{Q}}_{\delta} \underline{\underline{R}}_{\Delta \theta} \underline{\underline{F}}_{x y, q} \underline{\underline{u}}_{x y, p}^{T} \underline{\underline{T}}_{p q}^{T} \underline{\underline{R}}_{\Delta \theta}^{T} \underline{\underline{P}}_{\delta}^{T}\right)= \\
& =\frac{1}{2 R_{f} \delta} \sum_{p=1}^{m+1} \sum_{q=1}^{m+1} \operatorname{Tr}\left(\underline{F}_{x y, q} \underline{u}_{x y, p}^{T} \underline{\underline{T}}_{p q}^{T}\right) .
\end{aligned}
$$

As $G_{T O T}$ was defined according to Equation 12 and given that $\operatorname{Tr}(A B)=$ 
$\operatorname{Tr}(B A)$, it holds that

$$
\begin{aligned}
G_{T O T} & =\frac{1}{2 R_{f} \delta} \sum_{p=1}^{m+1} \sum_{q=1}^{m+1} \underline{u}_{r \theta, p}^{T} \underline{\underline{T}}_{p q}^{T} \underline{F}_{r \theta, q}=\frac{1}{2 R_{f} \delta} \sum_{p=1}^{m+1} \sum_{q=1}^{m+1} \operatorname{Tr}\left(\underline{F}_{r \theta, q} \underline{u}_{r \theta, p}^{T} \underline{\underline{T}}_{p q}^{T}\right)= \\
& =\frac{1}{2 R_{f} \delta} \sum_{p=1}^{m+1} \sum_{q=1}^{m+1} \operatorname{Tr}\left(\underline{F}_{x y, q} \underline{u}_{x y, p}^{T} \underline{\underline{T}}_{p q}^{T}\right)=\frac{1}{2 R_{f} \delta} \sum_{p=1}^{m+1} \sum_{q=1}^{m+1} \underline{u}_{x y, p}^{T} \underline{\underline{T}}_{p q}^{T} \underline{F}_{x y, q}
\end{aligned}
$$

190

which shows that the total Energy Release Rate is invariant to rigid rotations and can be calculated equivalently with forces and displacements expressed in the local crack tip reference frame or the global reference frame. The analytical result is confirmed by the numerical solution of the fiber-matrix interface crack with different element orders and model fiber volume fractions, as shown in 195 Figure 2,

The result of Equation 18 has also physical implications:

- given that stress and displacement fields at the crack tip are the same, two cracks with different crack paths are energetically equivalent with respect to the total Energy Release Rate:

- given that laws of the type $G_{T O T} \geq G_{c}$ govern crack propagation, if $G_{c}$ do not depend on mode ratio, crack orientation will not affect its growth. 

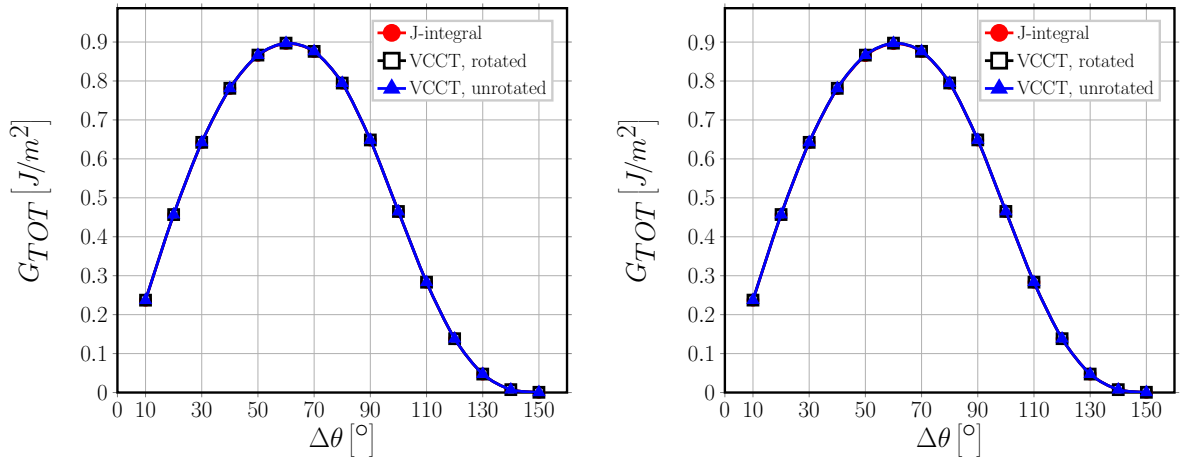

(a) $V_{f}=0.1 \%, 1^{s t}$ order elements, $\delta=$

(b) $V_{f}=0.1 \%, 2^{\text {nd }}$ order elements, $\delta=$ $0.05^{\circ}$.

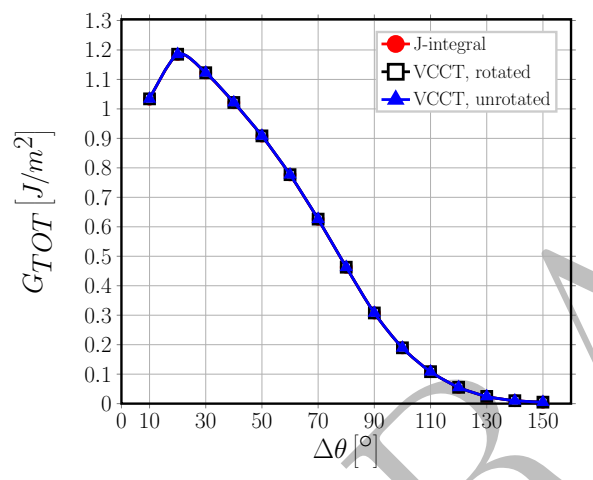

$0.05^{\circ}$

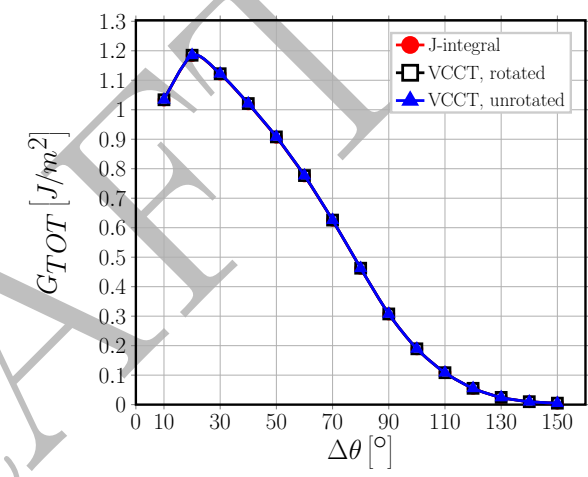

(c) $V_{f}=40 \%, 1^{\text {st }}$ order elements, $\delta=$

(d) $V_{f}=40 \%, 2^{\text {nd }}$ order elements, $\delta=$ $0.05^{\circ}$.

$0.05^{\circ}$.

Figure 2: Numerical invariance of the total Energy Release Rate: $G_{T O T}$ computed with the VCCT with rotated forces and displacements (label rotated), with the VCCT with forces and displacements in the global reference frame (label unrotated) and with J-integral method (label J-integral).

\section{Convergence analysis}

\subsection{Analytical considerations}

Substituting Equations 8 in the derivative of Equation 13 , we can investigate

the dependency of Mode I and Mode II ERR with respect to the size $\delta$ of an 
element in the crack tip neighborhood through

$$
\begin{aligned}
& \frac{\partial \underline{G}}{\partial \delta}=-\frac{1}{2 R_{f} \delta^{2}} \sum_{p=1}^{m+1} \sum_{q=1}^{m+1} \operatorname{Diag}\left(\underline{\underline{Q}}_{\delta} \underline{\underline{R}}_{\Delta \theta} \underline{\underline{K}}_{x y} \underline{u}_{x y} \underline{\underline{u}}_{x y}^{T} \underline{\underline{\underline{R}}}_{\Delta \theta}^{T} \underline{\underline{P}}_{\delta}^{T} \underline{\underline{T}}_{p q}^{T}\right)-\frac{1}{2 R_{f} \delta^{2}} \sum_{p=1}^{m+1} \sum_{q=1}^{m+1} \operatorname{Diag}\left(\underline{\underline{Q}}_{\delta} \underline{\underline{R}}_{\Delta \theta} \underline{\underline{\widetilde{K}}}_{N} \underline{\underline{u}}_{N} \underline{\underline{u}}_{x y}^{T} \underline{\underline{\underline{R}}}_{\Delta \theta}^{T} \underline{\underline{P}}_{\delta}^{T} \underline{\underline{T}}_{p q}^{T}\right)+ \\
& +\frac{1}{2 R_{f} \delta} \sum_{p=1}^{m+1} \sum_{q=1}^{m+1} \operatorname{Diag}\left(\underline{\underline{Q}}_{\delta} \underline{\underline{R}}_{\Delta \theta} \underline{\underline{K}}_{x y} \underline{\underline{u}}_{x y} \underline{\underline{u}}_{x y}^{T} \underline{\underline{R}}_{\Delta \theta}^{T} \underline{\underline{P}}_{\delta}^{T} \underline{\underline{D}}^{T} \underline{\underline{T}}_{p q}^{T}\right)+\frac{1}{2 R_{f} \delta} \sum_{p=1}^{m+1} \sum_{q=1}^{m+1} \operatorname{Diag}\left(\underline{\underline{Q}}_{\delta} \underline{\underline{R}}_{\Delta \theta} \underline{\underline{\underline{K}}}_{N} \underline{\underline{u}}_{N} \underline{\underline{u}}_{x y}^{T} \underline{\underline{R}}_{\Delta \theta}^{T} \underline{\underline{P}}_{\delta}^{T} \underline{\underline{D}}^{T} \underline{\underline{T}}_{p q}^{T}\right)+ \\
& +\frac{1}{2 R_{f} \delta} \sum_{p=1}^{m+1} \sum_{q=1}^{m+1} \operatorname{Diag}\left(\underline{\underline{D}} \underline{\underline{Q}} \underline{\underline{R}}_{\delta} \underline{\underline{R}}_{\Delta \theta} \underline{\underline{K}}_{x y} \underline{u}_{x y} \underline{u}_{x y}^{T} \underline{\underline{\underline{R}}}_{\Delta \theta}^{T} \underline{\underline{P}}_{\delta}^{T} \underline{\underline{T}}_{p q}^{T}\right)+\frac{1}{2 R_{f} \delta} \sum_{p=1}^{m+1} \sum_{q=1}^{m+1} \operatorname{Diag}\left(\underline{\underline{D}}_{\underline{\underline{Q}}} \underline{\underline{R}}_{\Delta \theta} \underline{\underline{\widetilde{K}}}_{N} \underline{\underline{u}}_{N} \underline{\underline{u}}_{x y}^{T} \underline{\underline{\underline{R}}}_{\Delta \theta}^{T} \underline{\underline{P}}_{\delta}^{T} \underline{\underline{T}}_{p q}^{T}\right)+ \\
& +\frac{1}{2 R_{f} \delta} \sum_{p=1}^{m+1} \sum_{q=1}^{m+1} \operatorname{Diag}\left(\underline{\underline{Q}}_{\delta} \underline{\underline{R}}_{\Delta \theta} \underline{\underline{K}}_{x y} \frac{\partial \underline{u}_{x y}}{\partial \delta} \underline{\underline{u}}_{x y}^{T} \underline{\underline{R}}_{\Delta \theta}^{T} \underline{\underline{P}}_{\delta}^{T} \underline{\underline{T}}_{p q}^{T}\right)+\frac{1}{2 R_{f} \delta} \sum_{p=1}^{m+1} \sum_{q=1}^{m+1} \operatorname{Diag}\left(\underline{\underline{Q}}_{\delta} \underline{\underline{R}}_{\Delta \theta} \underline{\underline{\underline{K}}}_{N} \frac{\partial \underline{\underline{u}}_{N}}{\partial \delta} \underline{\underline{u}}_{x y}^{T} \underline{\underline{R}}_{\Delta \theta}^{T} \underline{\underline{P}}_{\delta}^{T} \underline{\underline{T}}_{p q}^{T}\right)+ \\
& +\frac{1}{2 R_{f} \delta} \sum_{p=1}^{m+1} \sum_{q=1}^{m+1} \operatorname{Diag}\left(\underline{\underline{Q}}_{\delta} \underline{\underline{R}}_{\Delta \theta} \underline{\underline{K}}_{x y} \underline{\underline{u}}_{x y} \frac{\partial \underline{u}_{x y}^{T}}{\partial \delta} \underline{\underline{R}}_{\Delta \theta}^{T} \underline{\underline{P}}_{\delta}^{T} \underline{\underline{T}}_{p q}^{T}\right)+\frac{1}{2 R_{f} \delta} \sum_{p=1}^{m+1} \sum_{q=1}^{m+1} \operatorname{Diag}\left(\underline{\underline{Q}}_{\delta} \underline{\underline{R}}_{\Delta \theta} \underline{\underline{\widetilde{K}}}_{N} \underline{\underline{u}}_{N} \frac{\partial \underline{\underline{u}}_{x y}^{T}}{\partial \delta} \underline{\underline{R}}_{\Delta \theta}^{T} \underline{\underline{P}}_{\delta}^{T} \underline{\underline{T}}_{p q}^{T}\right) ;
\end{aligned}
$$

which, after refactoring, provides

$$
\begin{aligned}
& \frac{\partial \underline{G}}{\partial \delta}=\frac{1}{\delta} \underline{G}+\frac{1}{2 R_{f} \delta} \sum_{p=1}^{m+1} \sum_{q=1}^{m+1} \operatorname{Diag}\left(\underline{\underline{Q}}_{\delta} \underline{\underline{R}}_{\Delta \theta}\left(\underline{\underline{K}}_{x y} \underline{\underline{u}}_{x y}+\underline{\underline{\widetilde{K}}}_{N} \underline{u}_{N}\right) \underline{\underline{u}}_{x y}^{T} \underline{\underline{R}}_{\Delta \theta}^{T} \underline{\underline{P}}_{\delta}^{T} \underline{\underline{D}}^{T} \underline{\underline{T}}_{p q}^{T}\right)+ \\
& +\frac{1}{2 R_{f} \delta} \sum_{p=1}^{m+1} \sum_{q=1}^{m+1} \operatorname{Diag}\left(\underline{\underline{D}}_{\underline{\underline{Q}}} \underline{\underline{R}}_{\Delta \theta}\left(\underline{\underline{K}}_{x y} \underline{\underline{u}}_{x y}+\underline{\underline{\underline{K}}}_{N} \underline{\underline{u}}_{N}\right) \underline{\underline{u}}_{x y}^{T} \underline{\underline{R}}_{\Delta \theta}^{T} \underline{\underline{P}}_{\delta}^{T} \underline{\underline{T}}_{p q}^{T}\right)+
\end{aligned}
$$

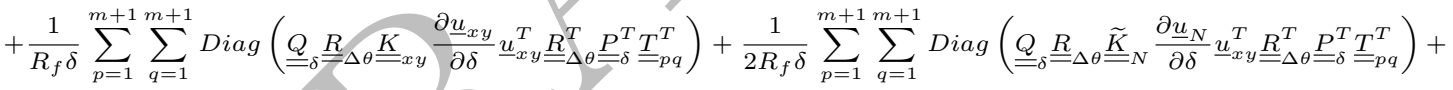

$$
\begin{aligned}
& +\frac{1}{2 R_{f} \delta} \sum_{p=1}^{m+1} \sum_{q=1}^{m+1} \operatorname{Diag}\left(\underline{\underline{Q}}_{\delta} \underline{\underline{R}} \Delta \theta \underline{\underline{K}}_{N} \underline{\underline{u}}_{N} \frac{\partial \underline{u}_{x y}^{T}}{\partial \delta} \underline{\underline{R}}_{\Delta \theta}^{T} \underline{\underline{P}}_{\delta}^{T} \underline{\underline{T}}_{p q}^{T}\right) \text {. }
\end{aligned}
$$

Following the asymptotic analysis of 3, 1, in the case of an open crack the displacement in the crack tip neighborhood will have a functional form of the type

$$
u(\delta) \sim \sqrt{\delta}(\sin , \cos )(\epsilon \log \delta) \quad \text { with } \quad \epsilon=\frac{1}{2 \pi} \log \left(\frac{1-\beta}{1+\beta}\right)
$$

and $\beta$ is Dundurs' parameter introduced in Section 1. Application of Equation 21 to the terms on the right hand side of Eq. 20 provides:

$$
\begin{gathered}
\underline{u}_{x y}, \underline{u}_{N} \sim u(\delta) \sim \sqrt{\delta}(\sin , \cos )(\epsilon \log \delta) \stackrel{\delta \rightarrow 0}{\longrightarrow} 0 \\
\underline{u}_{x y} \underline{u}_{x y}^{T}, \underline{u}_{N} \underline{u}_{x y}^{T} \sim u^{2}(\delta) \sim \delta\left(\sin ^{2}, \cos ^{2}, \sin \cdot \cos \right)(\epsilon \log \delta) \stackrel{\delta \rightarrow 0}{\longrightarrow} 0
\end{gathered}
$$


$\frac{\partial \underline{u}_{x y}}{\partial \delta} \underline{u}_{x y}^{T}, \frac{\partial \underline{u}_{N}}{\partial \delta} \underline{u}_{x y}^{T} \sim-\frac{1}{2}\left(\sin ^{2}, \cos ^{2}, \sin \cdot \cos \right)(\epsilon \log \delta)+\left(-\sin ^{2}, \cos ^{2}, \pm \sin \cdot \cos \right)(\epsilon \log \delta) \stackrel{\delta \rightarrow 0}{\longrightarrow}$ finite;

$$
\underline{G} \sim \frac{1}{\delta} \underline{u}_{x y} \underline{u}_{x y}^{T} \sim \frac{1}{\delta} u^{2}(\delta) \sim\left(\sin ^{2}, \cos ^{2}, \sin \cdot \cos \right)(\epsilon \log \delta) \stackrel{\delta \rightarrow 0}{\longrightarrow} \text { finite. }
$$

In Equations 22 25, the multiplication by a trigonometric function of the type $\left(\sin , \cos , \sin ^{2}, \cos ^{2}, \sin \cdot \cos \right)$ prevents the divergence of the asymptote. Recalling identity matrix:

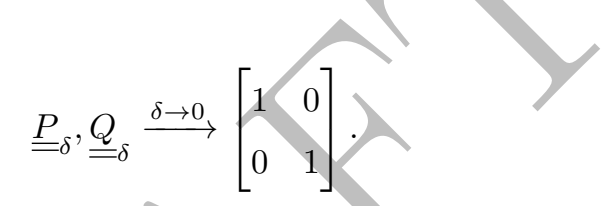

Applying the results of Equations 22,26 to Eq. 20 it can be shown that the derivative of $\underline{G}$ can be split in a factor that goes to 0 in the limit of $\delta \rightarrow 0$ and in a factor independent of $\delta$ :

$$
\lim _{\delta \rightarrow 0} \frac{\partial \underline{G}}{\partial \delta} \sim \frac{1}{\delta}\left(F(\delta)^{0}+\underline{C}\right) .
$$

Thus, asymptotically, the Mode I and Mode II Energy Release Rate behave like the logarithm of the angular size $\delta$ of the elements in the crack tip neighborhood:

$$
\lim _{\delta \rightarrow 0} \frac{\partial \underline{G}}{\partial \delta} \sim \frac{1}{\delta} \quad \stackrel{\int d \delta}{\longrightarrow} \quad \lim _{\delta \rightarrow 0} \underline{G} \sim \underline{A} \log (\delta)+\underline{B} .
$$

\subsection{Numerical results}

Evaluations of the Mode I, Mode II and total Energy Release Rate using the VCCT applied to the FE solution of the fiber-matrix interface crack in the single fiber model of Sec. 2 are reported respectively in Fig. 3 . Fig. 4 and Fig. 5

Results for Mode I ERR in Fig. 3 show clearly the transition from the open crack regime, where Mode I ERR is different from zero, to the closed crack 


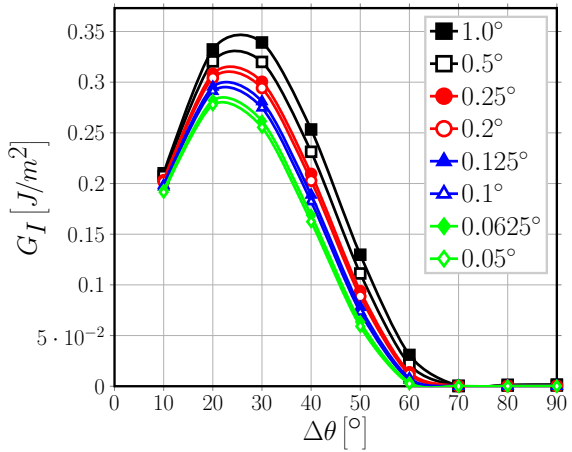

(a) $V_{f}=0.1 \%, 1^{s t}$ order elements.

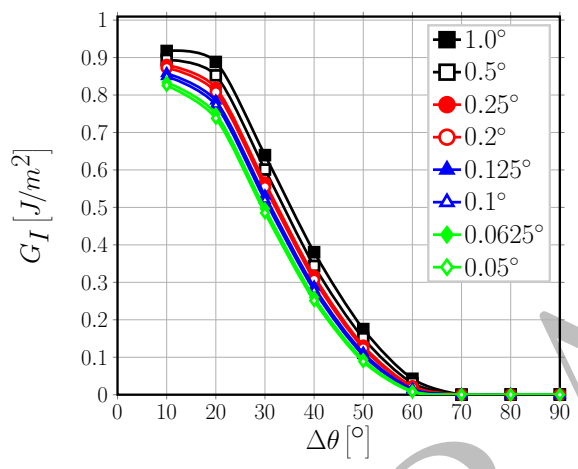

(c) $V_{f}=40 \%, 1^{\text {st }}$ order elements.

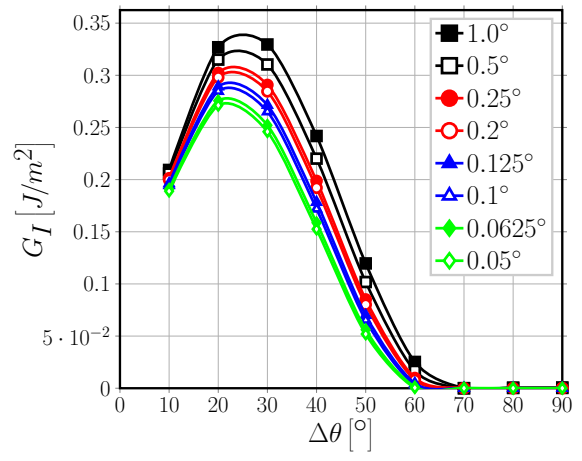

(b) $V_{f}=0.1 \%, 2^{\text {nd }}$ order elements.

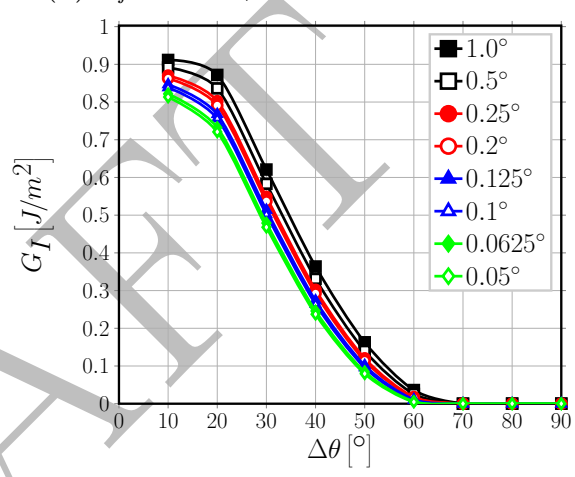

(d) $V_{f}=40 \%, 2^{\text {nd }}$ order elements.

Figure 3: Effect of the size $\delta$ of an element at the crack tip on Mode I ERR.

regime of the debond, where $G_{I}=0$. Looking at Fig. 3, the crack is open for $\Delta \theta \leq 60^{\circ}$ and it is closed, i.e. a contact zone is present, for $\Delta \theta \geq 70^{\circ}$. As expected from the analysis of the previous section, and given that Mode I ERR is different from zero only in the open crack regime, a significant dependence on the element size $\delta$ can be observed in Fig. 3 when using both $1^{\text {st }}$ and $2^{\text {nd }}$ order elements and with both an effectively infinite $\left(V_{f}=0.1 \%\right)$ and finite size $\left(V_{f}=40 \%\right)$ matrix. At first sight, it is immediate to see from Fig. 3 that a decrease in $\delta$ leads to a decrease in $G_{I}$. However, two further effects can be observed due to the refinement of the mesh at the crack tip, i.e. the decrease of the element size $\delta$. First, the occurrence of the peak $G_{I}$ is shifted to lower angles for very low volume fractions: it occurs at $\Delta \theta=30^{\circ}$ with $\delta=1.0^{\circ}, 0.5^{\circ}$ and at 
Second, the apperance of the contact zone, i.e. the switch to the closed crack regime, is anticipated to smaller debonds: it occurs at $\Delta \theta=70^{\circ}$ with $\delta \geq 0.2^{\circ}$ and at $\Delta \theta=60^{\circ}$ with $\delta<0.2^{\circ}$ for both $1^{\text {st }}$ and $2^{\text {nd }}$ order elements and both $V_{f}=0.1 \%$ and $V_{f}=40 \%$.

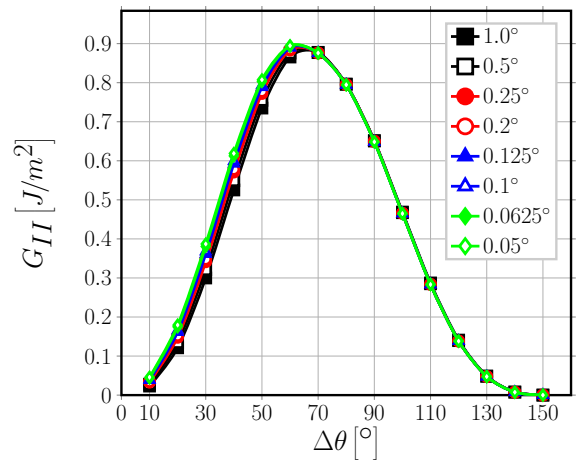

(a) $V_{f}=0.1 \%, 1^{s t}$ order elements.

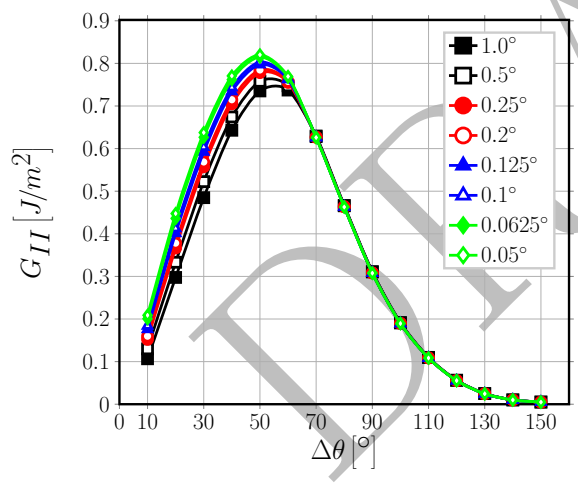

(c) $V_{f}=40 \%, 1^{\text {st }}$ order elements.

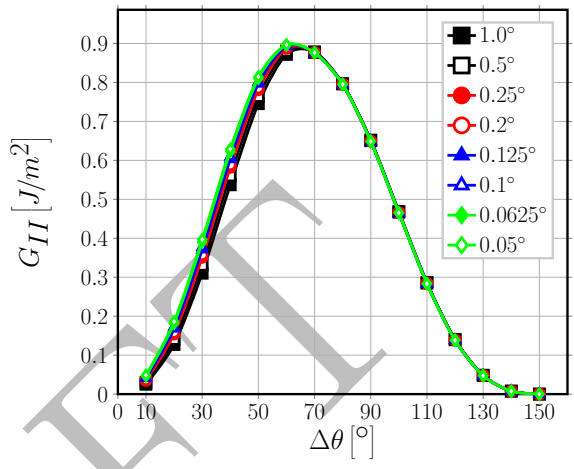

(b) $V_{f}=0.1 \%, 2^{\text {nd }}$ order elements.

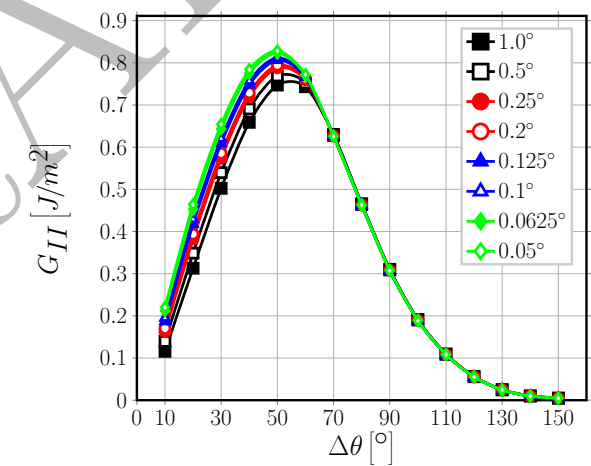

(d) $V_{f}=40 \%, 2^{\text {nd }}$ order elements.

Figure 4: Effect of the size $\delta$ of an element at the crack tip on Mode II ERR.

Observing Figure 4 it possible to notice the existence of two distinct regimes in the behavior of $G_{I I}$ with respect to the element size $\delta$. For $\Delta \theta \leq 60^{\circ} G_{I I}$ depends on the value of $\delta$, while $\Delta \theta \geq 70^{\circ}$ it is effectively independent of the element size at the crack tip for both $1^{\text {st }}$ and $2^{\text {nd }}$ order elements and both an effectively infinite $\left(V_{f}=0.1 \%\right)$ and finite size $\left(V_{f}=40 \%\right)$ matrix. Comparing the value of $\Delta \theta$ at which the change from the $\delta$-dependency regime to the $\delta$ - 
independency regime occurs for $G_{I I}$ with Mode I ERR in Fig. 3, it is possible to observe that the $\delta$-dependency regime change of Mode II ERR coincides with the onset of the contact zone, i.e. the transition from open crack regime to the closed crack regime. The result confirms the analytical considerations of the previous section: for an open crack both Mode I and Mode II ERR depend on the element size $\delta$ at the crack tip.
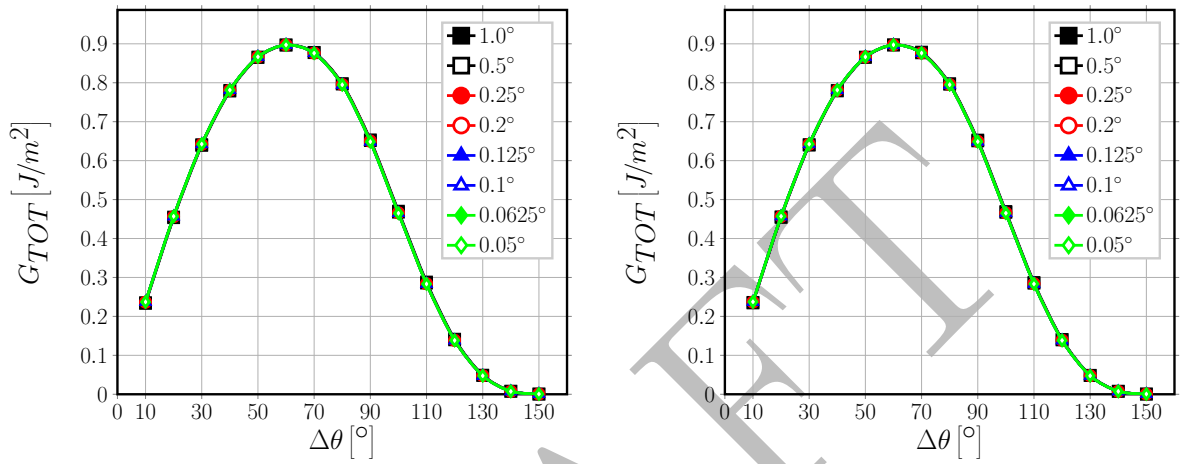

(a) $V_{f}=0.1 \%, 1^{s t}$ order elements.

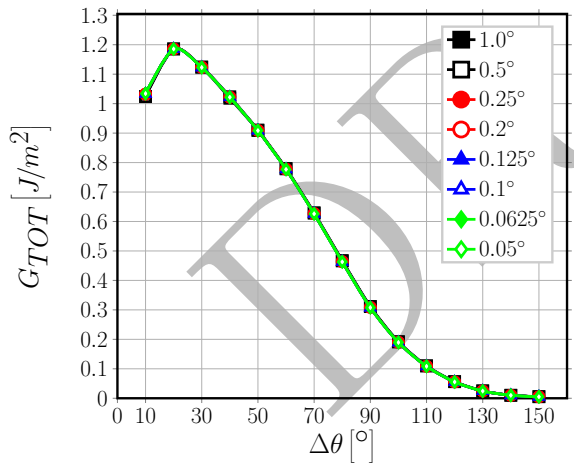

(b) $V_{f}=0.1 \%, 2^{\text {nd }}$ order elements.

(c) $V_{f}=40 \%, 1^{\text {st }}$ order elements.

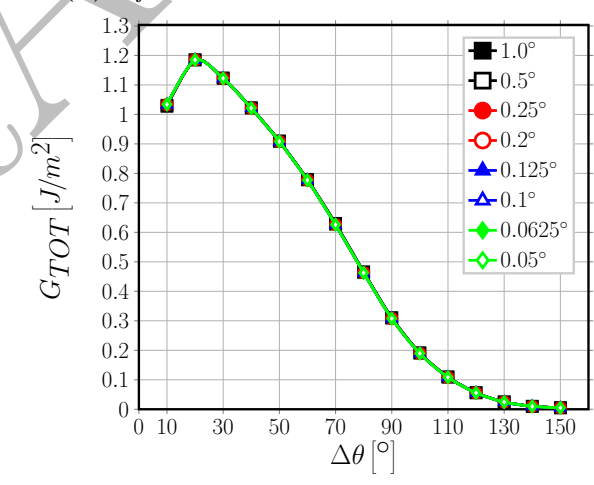

(d) $V_{f}=40 \%, 2^{\text {nd }}$ order elements.

Figure 5: Effect of the size $\delta$ of an element at the crack tip on total ERR.

Further observation of Figure 4 reveals that, in the open crack regime, decreasing the element size $\delta$ causes an increase of Mode II ERR. Similarly to Mode I ERR, a shift of the peak $G_{I I}$ can also observed for $V_{f}=0.1 \%$ : the maximum value of $G_{I I}$ occurs at $\Delta \theta=70^{\circ}$ for $\delta>0.25^{\circ}$ for $1^{\text {st }}$ order elements and for $\delta>0.5^{\circ}$ for $2^{\text {nd }}$ order elements, while it is shifted to $\Delta \theta=60^{\circ}$ for 
$\delta \leq 0.25^{\circ}$ for $1^{\text {st }}$ order elements and for $\delta \leq 0.5^{\circ}$ for $2^{\text {nd }}$ order elements.

Table 2: Summary of linear regression results and main statistical tests for Mode I ERR

\begin{tabular}{|c|c|c|c|c|c|c|c|c|}
\hline $\mathbf{V}_{\mathbf{f}}[\%]$ & Order & $\boldsymbol{\Delta} \theta\left[^{\circ}\right]$ & $\mathbf{A}\left[\frac{\mathrm{J}}{\mathbf{m}^{2}}\right]$ & $\mathbf{B}\left[\frac{\mathrm{J}}{\mathrm{m}^{2}}\right]$ & $\mathbf{r}[-]$ & $\mathbf{r}^{2}[-]$ & $\mathbf{p}(\mathbf{A})[-]$ & $\mathbf{p}(\mathbf{B})[-]$ \\
\hline \multirow[t]{6}{*}{0.1} & 1 & 10.0 & 0.0064 & 0.2113 & 0.9933 & 0.9866 & $7.48 \mathrm{E}-07$ & $3.49 \mathrm{E}-14$ \\
\hline & & 20.0 & 0.0183 & 0.3331 & 0.9996 & 0.9992 & $1.44 \mathrm{E}-10$ & $2.40 \mathrm{E}-16$ \\
\hline & & 30.0 & 0.0280 & 0.3392 & 1.0000 & 1.0000 & $2.25 \mathrm{E}-16$ & $4.26 \mathrm{E}-21$ \\
\hline & & 40.0 & 0.0304 & 0.2524 & 0.9997 & 0.9995 & $4.38 \mathrm{E}-11$ & $7.94 \mathrm{E}-15$ \\
\hline & & 50.0 & 0.0235 & 0.1278 & 0.9985 & 0.9970 & 8.61E-09 & $2.01 \mathrm{E}-11$ \\
\hline & & 60.0 & 0.0094 & 0.0284 & 0.98 & 0.9709 & $7.75 \mathrm{E}-06$ & $6.14 \mathrm{E}-07$ \\
\hline \multirow[t]{6}{*}{0.1} & 2 & 10.0 & 0.0069 & 0.2103 & & 0.9 & $1.36 \mathrm{E}-07$ & $1.03 \mathrm{E}-14$ \\
\hline & & 20.0 & 0.0187 & 0.3277 & 0.9997 & 0.9994 & $7.85 \mathrm{E}-11$ & $1.62 \mathrm{E}-16$ \\
\hline & & 30.0 & 0.0280 & 0.3296 & & 1.0000 & $3.28 \mathrm{E}-16$ & $7.29 \mathrm{E}-21$ \\
\hline & & 40.0 & 0.0298 & 0.2408 & 0.9997 & 0.9995 & $4.82 \mathrm{E}-11$ & $1.04 \mathrm{E}-14$ \\
\hline & & 50.0 & 0.0225 & 0.1177 & 0.9 & 0.9967 & $1.10 \mathrm{E}-08$ & $3.27 \mathrm{E}-11$ \\
\hline & & 60.0 & 0.0081 & 0.0228 & 0.9811 & 0.9626 & $1.66 \mathrm{E}-05$ & $2.17 \mathrm{E}-06$ \\
\hline \multirow[t]{6}{*}{40} & 1 & 10.0 & & 0.9196 & 0.9963 & 0.9927 & $1.03 \mathrm{E}-07$ & $9.33 \mathrm{E}-15$ \\
\hline & & & 0501 & 0.8882 & 1.0000 & 0.9999 & $1.21 \mathrm{E}-13$ & $2.33 \mathrm{E}-19$ \\
\hline & & 30.0 & 0.0510 & 0.6374 & 0.9998 & 0.9996 & $1.66 \mathrm{E}-11$ & $2.58 \mathrm{E}-16$ \\
\hline & & & 0.0419 & 0.3760 & 0.9988 & 0.9976 & $4.56 \mathrm{E}-09$ & $5.25 \mathrm{E}-13$ \\
\hline & & 50.0 & 0.0279 & 0.1713 & 0.9980 & 0.9961 & $2.22 \mathrm{E}-08$ & $2.52 \mathrm{E}-11$ \\
\hline & & 60.0 & 0.0108 & 0.0391 & 0.9901 & 0.9804 & $3.44 \mathrm{E}-06$ & $9.46 \mathrm{E}-08$ \\
\hline \multirow[t]{6}{*}{40} & 2 & 10.0 & 0.0336 & 0.9148 & 0.9988 & 0.9977 & $3.45 \mathrm{E}-09$ & $5.09 \mathrm{E}-16$ \\
\hline & & 20.0 & 0.0504 & 0.8719 & 1.0000 & 1.0000 & $3.70 \mathrm{E}-14$ & $8.26 \mathrm{E}-20$ \\
\hline & & 30.0 & 0.0506 & 0.6191 & 0.9999 & 0.9997 & $7.63 \mathrm{E}-12$ & $1.35 \mathrm{E}-16$ \\
\hline & & 40.0 & 0.0414 & 0.3608 & 0.9994 & 0.9989 & $4.95 \mathrm{E}-10$ & $6.80 \mathrm{E}-14$ \\
\hline & & 50.0 & 0.0269 & 0.1593 & 0.9982 & 0.9964 & $1.66 \mathrm{E}-08$ & $2.31 \mathrm{E}-11$ \\
\hline & & 60.0 & 0.0097 & 0.0329 & 0.9890 & 0.9781 & $4.96 \mathrm{E}-06$ & $1.99 \mathrm{E}-07$ \\
\hline
\end{tabular}

Analysis of the total ERR in Figure 5 leads to an observation that was 
not predicted by the considerations of the previous section: $G_{T O T}$ is effectively independent of the element size $\delta$ in both the open and the closed crack regimes, at least for reasonably small elements $\left(\delta \leq 1.0^{\circ}\right)$. Given that $G_{I I}=G_{T O T}$ for the closed crack, it explains the independency of $G_{I I}$ from $\delta$ after the onset of the contact zone.

Analysis of Fig. 3, Fig. 4 and Fig. 5 has shown the dependency of Mode I and Mode II ERR on the element size $\delta$. Following the derivations of the previous section, we model the dependency of $G_{I}$ and $G_{I I}$ with respect to $\delta$ as

$$
G_{(\cdot)}=A(\Delta \theta) \ln \delta+B(\Delta \theta)
$$

where $A(\Delta \theta)$ and $B(\Delta \theta)$ are parameters dependent on $\Delta \theta$ estimated through linear regression (with $x=\ln \delta$ ) of the numerical results.

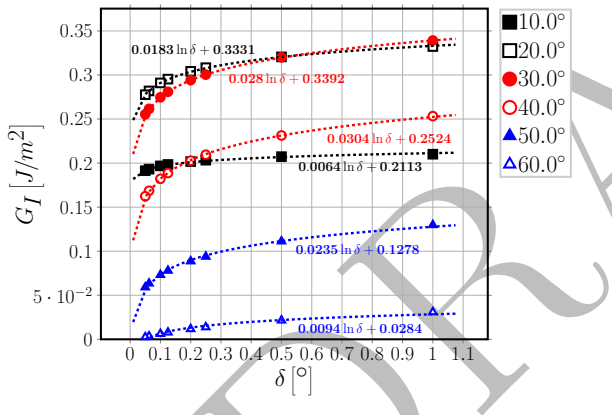

(a) $1^{\text {st }}$ order elements.

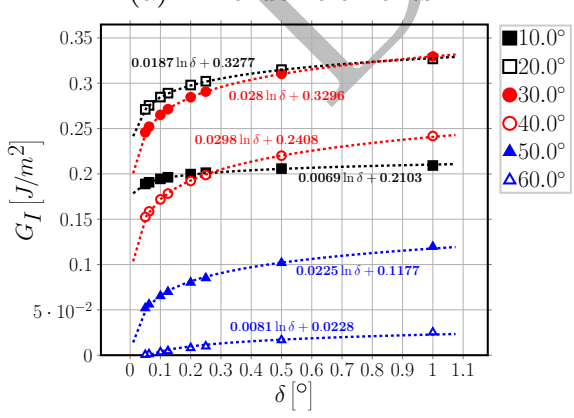

(c) $2^{\text {nd }}$ order elements.

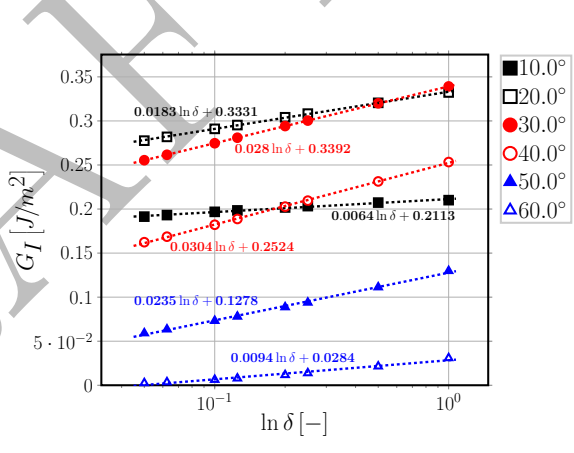

(b) $1^{\text {st }}$ order elements.

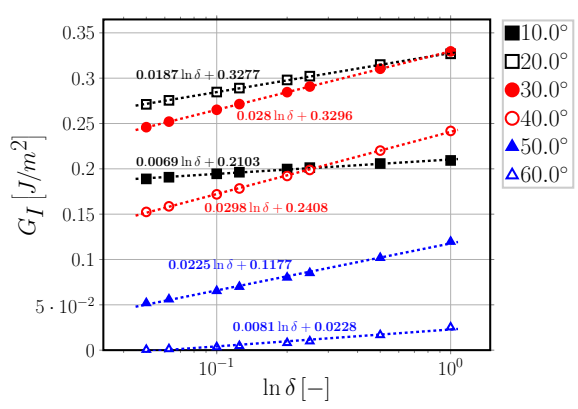

(d) $2^{\text {nd }}$ order elements.

Figure 6: Logarithmic dependence on $\delta$ of Mode I ERR: interpolation of numerical results for $V_{f}=0.1 \%$. 
Table 3: Summary of linear regression results and main statistical tests for Mode II ERR

\begin{tabular}{|c|c|c|c|c|c|c|c|c|}
\hline $\mathbf{V}_{\mathbf{f}}[\%]$ & Order & $\boldsymbol{\Delta} \theta\left[^{\circ}\right]$ & $\mathbf{A}\left[\frac{\mathrm{J}}{\mathrm{m}^{2}}\right]$ & $\mathrm{B}\left[\frac{\mathrm{J}}{\mathrm{m}^{2}}\right]$ & $\mathbf{r}[-]$ & $\mathbf{r}^{2}[-]$ & $\mathbf{p}(\mathbf{A})[-]$ & $\mathbf{p}(\mathbf{B})[-]$ \\
\hline \multirow[t]{6}{*}{0.1} & 1.0 & 10.0 & -0.0076 & 0.0228 & -0.9996 & 0.9991 & $2.09 \mathrm{E}-10$ & $1.64 \mathrm{E}-11$ \\
\hline & & 20.0 & -0.0194 & 0.1211 & -1.0000 & 1.0000 & $1.99 \mathrm{E}-15$ & $2.02 \mathrm{E}-18$ \\
\hline & & 30.0 & -0.0290 & 0.3007 & -0.9999 & 0.9998 & $4.12 \mathrm{E}-12$ & $1.97 \mathrm{E}-16$ \\
\hline & & 40.0 & -0.0311 & 0.5270 & -0.9995 & 0.9989 & $4.13 \mathrm{E}-10$ & $1.05 \mathrm{E}-15$ \\
\hline & & 50.0 & -0.0240 & 0.7375 & -0.9979 & 0.9958 & $2.32 \mathrm{E}-08$ & $1.66 \mathrm{E}-15$ \\
\hline & & 60.0 & -0.0095 & 0.8685 & -0.9835 & 0.9672 & $1.12 \mathrm{E}-05$ & $1.22 \mathrm{E}-15$ \\
\hline \multirow[t]{6}{*}{0.1} & 2.0 & 10.0 & -0.0078 & 0.0249 & & 0.9992 & $1.91 \mathrm{E}-10$ & $1.06 \mathrm{E}-11$ \\
\hline & & 20.0 & -0.0196 & 0.1272 & 00 & 1.0000 & $3.48 \mathrm{E}-15$ & $2.78 \mathrm{E}-18$ \\
\hline & & 30.0 & -0.0288 & 0.3 & & 0.9998 & $1.45 \mathrm{E}-12$ & $5.47 \mathrm{E}-17$ \\
\hline & & 40.0 & -0.0305 & 0.5387 & 93 & 0.9990 & $3.32 \mathrm{E}-10$ & $6.55 \mathrm{E}-16$ \\
\hline & & 50.0 & -0.0229 & 0.7478 & -0.9979 & 0.9959 & $2.17 \mathrm{E}-08$ & $1.09 \mathrm{E}-15$ \\
\hline & & 60.0 & -0.0082 & 0.8 & -0.9806 & 0.9615 & $1.81 \mathrm{E}-05$ & $8.26 \mathrm{E}-16$ \\
\hline \multirow[t]{6}{*}{40.0} & 1.0 & 10.0 & & 0.1055 & -0.9997 & 0.9995 & $3.82 \mathrm{E}-11$ & $2.73 \mathrm{E}-12$ \\
\hline & & 20. & & 0.2 & -1.0000 & 0.9999 & $4.22 \mathrm{E}-14$ & $5.66 \mathrm{E}-17$ \\
\hline & & & & 0.4866 & -0.9999 & 0.9997 & $6.44 \mathrm{E}-12$ & $4.82 \mathrm{E}-16$ \\
\hline & & 40.0 & & 0.6454 & -0.9996 & 0.9991 & $2.12 \mathrm{E}-10$ & $9.66 \mathrm{E}-16$ \\
\hline & & 50.0 & -0.0275 & 0.7386 & -0.9985 & 0.9971 & $9.01 \mathrm{E}-09$ & $1.44 \mathrm{E}-15$ \\
\hline & & & -0.0099 & 0.7402 & -0.9926 & 0.9853 & $1.41 \mathrm{E}-06$ & $5.13 \mathrm{E}-16$ \\
\hline \multirow[t]{6}{*}{40.0} & 2.0 & 10.0 & -0.0353 & 0.1145 & -0.9998 & 0.9995 & $2.92 \mathrm{E}-11$ & $1.50 \mathrm{E}-12$ \\
\hline & & 20.0 & -0.0504 & 0.3130 & -1.0000 & 0.9999 & $4.00 \mathrm{E}-14$ & $4.17 \mathrm{E}-17$ \\
\hline & & 30.0 & -0.0502 & 0.5039 & -0.9999 & 0.9998 & $2.87 \mathrm{E}-12$ & $1.69 \mathrm{E}-16$ \\
\hline & & 40.0 & -0.0410 & 0.6615 & -0.9996 & 0.9992 & $2.02 \mathrm{E}-10$ & $6.89 \mathrm{E}-16$ \\
\hline & & 50.0 & -0.0263 & 0.7502 & -0.9987 & 0.9973 & $6.87 \mathrm{E}-09$ & $7.76 \mathrm{E}-16$ \\
\hline & & 60.0 & -0.0090 & 0.7458 & -0.9921 & 0.9842 & $1.79 \mathrm{E}-06$ & $3.37 \mathrm{E}-16$ \\
\hline
\end{tabular}

As shown in Fig. 6, Fig. 7 Fig. 8 and Fig. 9 both in linear and logarithmic 275 scales of $\delta$, the result is remarkable: both the correlation coefficient $r$ and the 


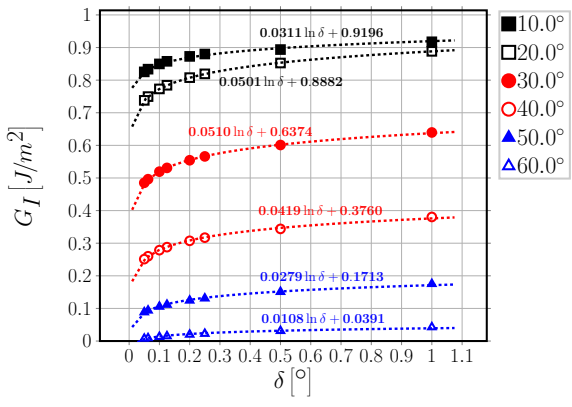

(a) $1^{\text {st }}$ order elements.

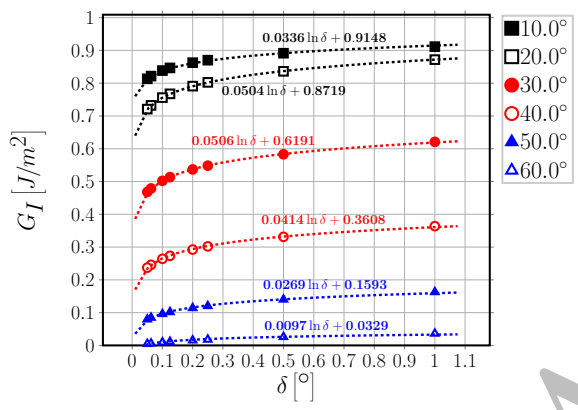

(c) $2^{\text {nd }}$ order elements.

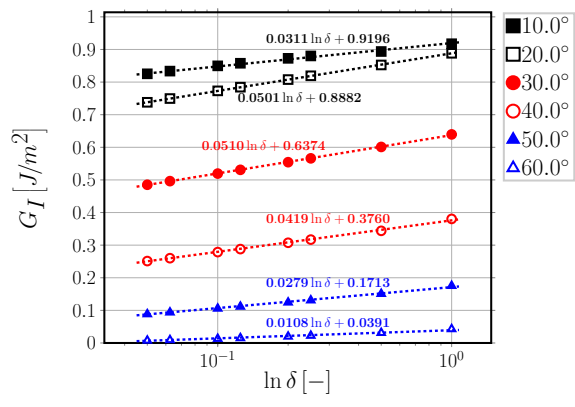

(b) $1^{\text {st }}$ order elements.

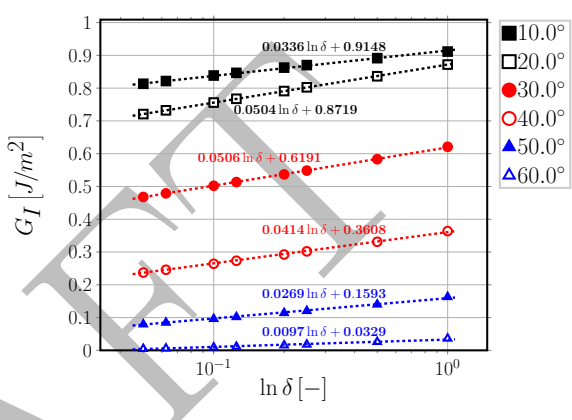

(d) $2^{\text {nd }}$ order elements.

Figure 7: Logarithmic dependence on $\delta$ of Mode I ERR: interpolation of numerical results for $V_{f}=40 \%$.

$r^{2}$ ratio (of explained to total variance) are always greater than 0.95 and the $p$-values of the coefficients $A$ and $B$ are at least $<1 E-6$ and often $<1 E-11$ (see Table 2 for $G_{I}$ and Table 3 for $G_{I I}$ ). The results of the linear regression confirm the analytical derivations of the previous section, which showed the logarithmic behavior of Mode I and Mode II ERR. Similar conclusions were reached in [28, 29] for a straight bi-material crack with respect to the parameter $\Delta a / a$; however, no functional expression of $G_{(\cdot)}$ was proposed. 


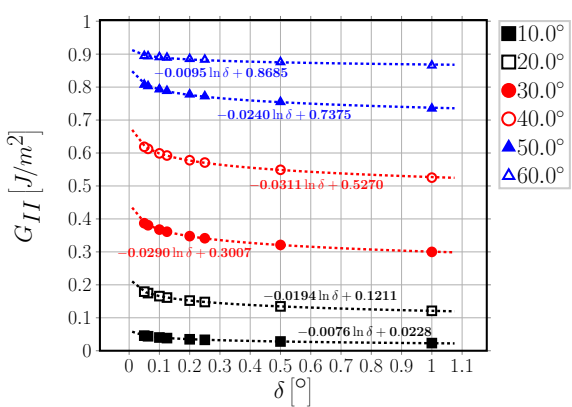

(a) $1^{\text {st }}$ order elements.

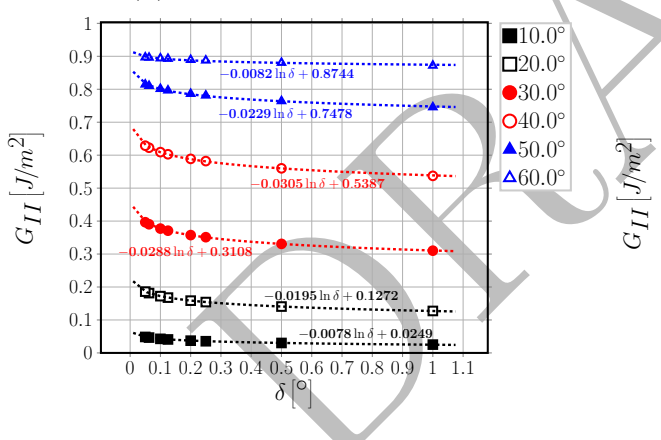

(c) $2^{\text {nd }}$ order elements.

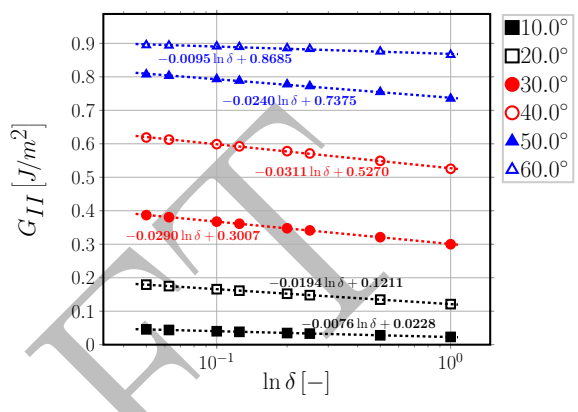

(b) $1^{\text {st }}$ order elements.

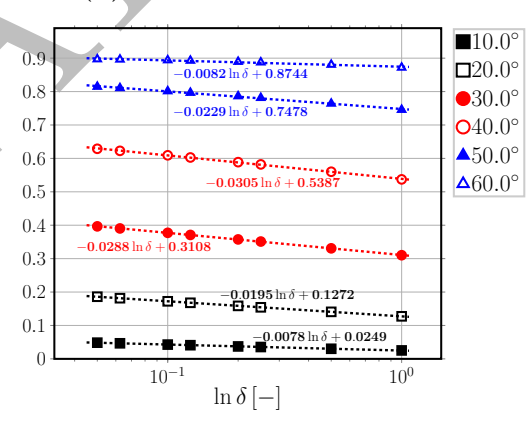

(d) $2^{\text {nd }}$ order elements.

Figure 8: Logarithmic dependence on $\delta$ of Mode II ERR: interpolation of numerical results for $V_{f}=0.1 \%$. 


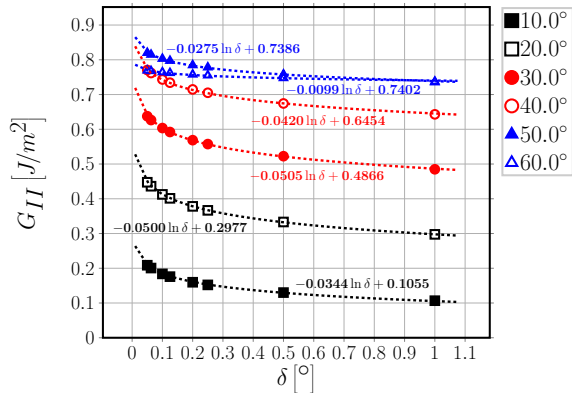

(a) $1^{\text {st }}$ order elements.

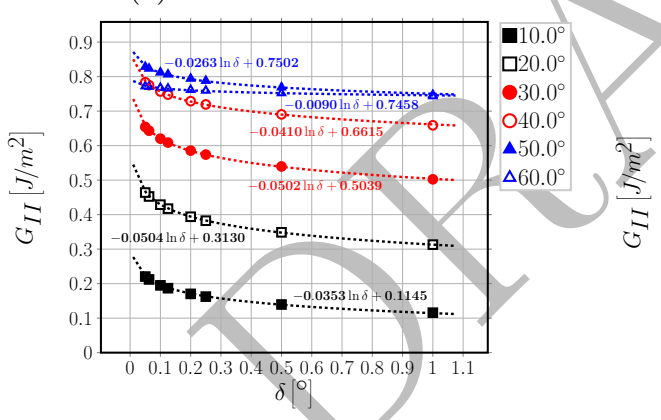

(c) $2^{\text {nd }}$ order elements.

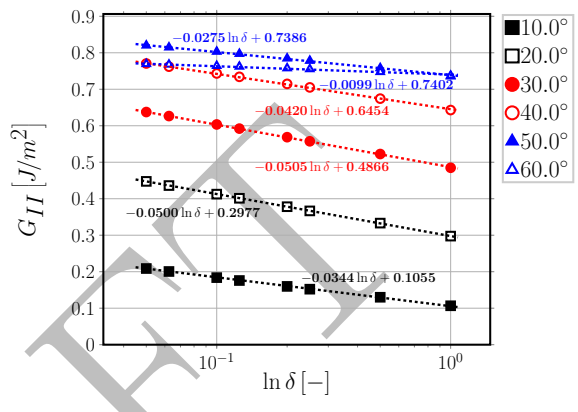

(b) $1^{\text {st }}$ order elements.

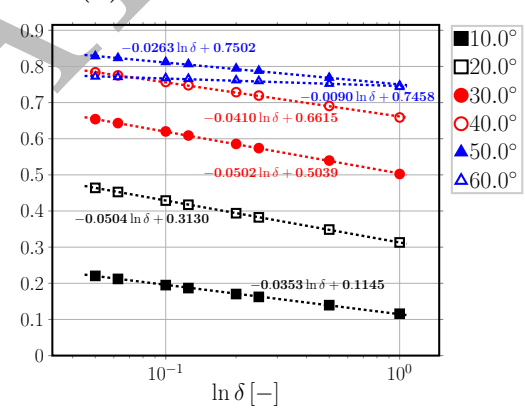

(d) $2^{\text {nd }}$ order elements.

Figure 9: Logarithmic dependence on $\delta$ of Mode II ERR: interpolation of numerical results for $V_{f}=40 \%$. 


\section{Conclusions \& Outlook}

The application of the Virtual Crack Closure Technique to the calculation of the Finite Element solution of the bi-material circular arc crack, or fibermatrix interface crack. A synthetic vectorial formulation of the VCCT has been proposed and its usefulness exemplified in the analysis of the mesh dependency. By both analytical considerations and numerical simulations, it has been shown that:

- the total ERR is invariant to rotations of the reference frame (and more in general to linear transformations), which implies that rotation of crack tip forces and displacement is actually not required in the use of the VCCT for the calculation of $G_{T O T}$;

- the total ERR does not depend on the size $\delta$ of the elements at the crack tip, at least for reasonably small elements $\left(\delta \leq 1.0^{\circ}\right)$;

- as a consequence, Mode II ERR for the closed interface crack does not depend on $\delta$, as $G_{I I}=G_{T O T}$ after the onset of the contact zone;

- for the open interface crack, Mode I and Mode II ERR depend on the element size $\delta$ through a logarithmic law of the type $A(\Delta \theta) \ln \delta+B(\Delta \theta)$;

- the sign of the logarithm is always positive for $G_{I}$, i.e. it decreases when $\delta$ decreases, and negative for $G_{I I}$, i.e. it increases when $\delta$ decreases.

The conclusion is significant: as the behavior of Mode I and Mode II is logarithmic with respect to mesh size, there exists no asymptotic limit and thus no convergence of the values. A convergence analysis based on the reduction of the error between successive iterations would not provide a reliable assessment of the accuracy of the FE solution of Mode I and Mode II Energy Release Rate of the fiber-matrix interface crack. A validation is thus required with respect to data obtained through a different method, be it analytical, numerical or experimental. Moreover, it has been shown that: first, the same behavior appears when 
using $1^{\text {st }}$ as well as $2^{\text {nd }}$ order elements; second, no improvement is expected with the use of singular elements, as the logarithmic dependency of $G_{I}$ and $G_{I I}$ is governed by the definition of ERR itself together with the asymptotic behavior of the displacement field at the crack tip. These two conclusions run contrary to the suggestions provided in the manuals of many commercial FEM packages, such as Abaqus [31] which suggests that (Section 11.4.2 of the Abaqus Analysis User's Guide): "Sharp cracks (where the crack faces lie on top of one another in the undeformed configuration) are usually modeled using small-strain assumptions. Focused meshes, [...], should normally be used for small-strain fracture mechanics evaluations. However, for a sharp crack the strain field becomes singular at the crack tip. [...] In most cases the singularity at the crack tip should be considered in small-strain analysis (when geometric nonlinearities are ignored). Including the singularity often improves the accuracy of the J-integral, the stress intensity factors, and the stress and strain calculations because the stresses and strains in the region close to the crack tip are more accurate.". We have shown that, in the context of the fiber/matrix interface crack, the convergence of the Energy Release Rate is determined by the asymptotic behavior of the elastic solution and only marginally by the choice of element order and type, thus contradicting the statements in 31 .

\section{Acknowledgements}

Luca Di Stasio thanks Prof. Janis Varna for the useful discussions and suggestions. Luca Di Stasio gratefully acknowledges the support of the European School of Materials (EUSMAT) through the DocMASE Doctoral Programme and the European Commission through the Erasmus Mundus Programme.

\section{References}

[1] M. Comninou, An overview of interface cracks, Engineering Fracture Mechanics 37 (1) (1990) 197-208. doi:10.1016/0013-7944(90)90343-f. 
[2] D. Hills, J. Barber, Interface cracks, International Journal of Mechanical Sciences 35 (1) (1993) 27-37. doi:10.1016/0020-7403(93)90062-y.

[3] M. L. Williams, The stresses around a fault or crack in dissimilar media, Bulletin of the Seismological Society of America 49 (2) (1959) 199.

[4] J. Dundurs, Discussion: "edge-bonded dissimilar orthogonal elastic wedges under normal and shear loading" (bogy, d. b., 1968, ASME j. appl. mech., 35, pp. 460-466), Journal of Applied Mechanics 36 (3) (1969) 650. doi: $10.1115 / 1.3564739$

[5] F. Erdogan, Stress distribution in a nonhomogeneous elastic plane with cracks, Journal of Applied Mechanics 30 (2) (1963) 232. doi:10.1115/1. 3636517 .

[6] A. H. England, A crack between dissimilar media, Journal of Applied Mechanics 32 (2) (1965) 400. doi:10.1115/1.3625813

[7] B. Malyshev, R. Salganik, The strength of adhesive joints using the theory

n. of cracks, International Journal of Fracture Mechanics 1-1 (2). doi:10. $1007 / \mathrm{bf} 00186749$.

URL https://doi.org/10.1007/bf00186749

355 [8] M. Comninou, The interface crack, Journal of Applied Mechanics 44 (4) (1977) 631. doi:10.1115/1.3424148

URL https://doi.org/10.1115/1.3424148

[9] A. H. England, An arc crack around a circular elastic inclusion, Journal of Applied Mechanics 33 (3) (1966) 637. doi:10.1115/1.3625132.

[10] A. Perlman, G. Sih, Elastostatic problems of curvilinear cracks in bonded dissimilar materials, International Journal of Engineering Science 5 (11) (1967) 845-867. doi:10.1016/0020-7225(67)90009-2.

[11] M. Toya, A crack along the interface of a circular inclusion embedded in an infinite solid, Journal of the Mechanics and Physics of Solids 22 (5) (1974) 325-348. doi:10.1016/0022-5096(74) 90002-7. 
[12] F. París, J. C. Caño, J. Varna, The fiber-matrix interface crack - a numerical analysis using boundary elements, International Journal of Fracture 82 (1) (1996) 11-29. doi:10.1007/bf00017861.

[13] G. R. Irwin, Fracture, in: Elasticity and Plasticity / Elastizität und Plastizität, Springer Berlin Heidelberg, 1958, pp. 551-590. doi:10.1007/ 978-3-642-45887-3_5.

[14] J. C. D. Caño, F. París, On stress singularities induced by the discretization in curved receding contact surfaces: a bem analysis, International Journal for Numerical Methods in Engineering 40 (12) (1997) aid-nme166>3.0.co;2-8.

[15] J. Varna, F. París, J. C.Caño, The effect of crack-face contact on fiber/matrix debonding in transverse tensile loading, Composites Science and Technology 57 (5) (1997) 523-532. doi:10.1016/s0266-3538(96) $380 \quad 00175-3$

[16] F. París, E. Correa, V. Mantič, Kinking of transversal interface cracks between fiber and matrix, Journal of Applied Mechanics 74 (4) (2007) 703. doi:10.1115/1.2711220.

[17] E. Correa, E. Gamstedt, F. París, V. Mantič, Effects of the presence of compression in transverse cyclic loading on fibre-matrix debonding in unidirectional composite plies, Composites Part A: Applied Science and Manufacturing 38 (11) (2007) 2260-2269. doi:10.1016/j.compositesa.2006. 11.002

[18] E. Correa, V. Mantič, F. París, Effect of thermal residual stresses on matrix failure under transverse tension at micromechanical level: A numerical and experimental analysis, Composites Science and Technology 71 (5) (2011) 622-629. doi:10.1016/j.compscitech.2010.12.027. 
[19] E. Correa, F. París, V. Mantič, Effect of the presence of a secondary transverse load on the inter-fibre failure under tension, Engineering Fracture Mechanics 103 (2013) 174-189. doi:10.1016/j.engfracmech.2013.02.026

[20] E. Correa, F. París, V. Mantič, Effect of a secondary transverse load on the inter-fibre failure under compression, Composites Part B: Engineering 65 (2014) 57-68. doi:10.1016/j.compositesb.2014.01.005

[21] C. Sandino, E. Correa, F. París, Numerical analysis of the influence of a nearby fibre on the interface crack growth in composites under transverse tensile load, Engineering Fracture Mechanics 168 (2016) 58-75. doi:10. 1016/j.engfracmech.2016.01.022.

[22] C. Sandino, E. Correa, F. París, Interface crack growth under transverse compression: nearby fibre effect, in: Proceeding of the $18^{\text {th }}$ European Conference on Composite Materials (ECCM-18), 2018.

[23] L. Zhuang, A. Pupurs, J. Varna, R. Talreja, Z. Ayadi, Effects of inter-fiber spacing on fiber-matrix debond crack growth in unidirectional composites under transverse loading, Composites Part A: Applied Science and Man口 ufacturing 109 (2018) 463-471. doi:10.1016/j.compositesa.2018.03.

[24] J. Varna, L. Q. Zhuang, A. Pupurs, Z. Ayadi, Growth and interaction of debonds in local clusters of fibers in unidirectional composites during transverse loading, Key Engineering Materials 754 (2017) 63-66. doi: 10.4028/www.scientific.net/kem.754.63

[25] L. Zhuang, R. Talreja, J. Varna, Transverse crack formation in unidirectional composites by linking of fibre/matrix debond cracks, Composites Part A: Applied Science and Manufacturing 107 (2018) 294-303. doi:10.1016/j.compositesa.2018.01.013

[26] E. Rybicki, M. Kanninen, A finite element calculation of stress intensity 
factors by a modified crack closure integral, Engineering Fracture Mechanics 9 (4) (1977) 931-938. doi:10.1016/0013-7944(77)90013-3.

[27] R. Krueger, Virtual crack closure technique: History, approach, and applications, Applied Mechanics Reviews 57 (2) (2004) 109. doi:10.1115/1. 1595677.

[28] C. Sun, C. Jih, On strain energy release rates for interfacial cracks in bimaterial media, Engineering Fracture Mechanics 28 (1) (1987) 13-20. doi: 10.1016/0013-7944(87)90115-9.

[29] M. Manoharan, C. Sun, Strain energy release rates of an interfacial crack between two anisotropic solids under uniform axial strain, Composites Science and Technology 39 (2) (1990) 99-116. doi:10.1016/0266-3538(90) 90049-b

[30] C. Sun, W. Qian, The use of finite extension strain energy release rates in fracture of interfacial cracks, International Journal of Solids and Structures 34 (20) (1997) 2595-2609. doi:10.1016/s0020-7683(96)00157-6.

${ }_{435}$ [31] Simulia, Providence, RI, USA, ABAQUS/Standard User's Manual, Version $6.12(2012)$.

[32] P. S. Valvo, A revised yirtual crack closure technique for physically consistent fracture mode partitioning, International Journal of Fracture 173 (1) (2011) 1-20. doi:10.1007/s10704-011-9658-y.

[33] I. Raju, Calculation of strain-energy release rates with higher order and singular finite elements, Engineering Fracture Mechanics 28 (3) (1987) 251274. doi:10.1016/0013-7944(87)90220-7. 


\section{Appendix A. Derivation of the relationship between crack tip forces and displacements for first order quadrilateral elements}

In the isoparametric formulation of the Finite Element Method, the element Jacobian $J$ and its inverse $J^{-1}$ can be expressed in general as

$$
\underline{\underline{J}}=\left[\underline{e}_{\xi} \mid \underline{e}_{\eta}\right]=\left[\begin{array}{ll}
\frac{\partial x}{\partial \xi} & \frac{\partial x}{\partial \eta} \\
\frac{\partial y}{\partial \xi} & \frac{\partial y}{\partial \eta}
\end{array}\right] \quad \underline{J}^{-1}=\left[\underline{e}^{x} \mid \underline{e}^{y}\right]=\left[\begin{array}{ll}
\frac{\partial \xi}{\partial x} & \frac{\partial \xi}{\partial y} \\
\frac{\partial \eta}{\partial x} & \frac{\partial \eta}{\partial y}
\end{array}\right]
$$

where $\left\{e_{\xi}, e_{\eta}\right\}$ and $\left\{e^{x}, e^{y}\right\}$ are respectively the covariant and contravariant basis vectors of the mapping between global $\{x, y\}$ and local element $\{\xi, \eta\}$ coordinates:

$$
\begin{gathered}
\underline{e}_{\xi}=\left[\begin{array}{c}
\frac{\partial x}{\partial \xi} \\
\frac{\partial y}{\partial \xi}
\end{array}\right] \quad \underline{e}_{\eta}=\left[\begin{array}{c}
\frac{\partial x}{\partial \eta} \\
\frac{\partial y}{\partial \eta}
\end{array}\right], \\
\underline{e}_{x}=\left[\begin{array}{c}
\frac{\partial \xi}{\partial x} \\
\frac{\partial \eta}{\partial x}
\end{array}\right] \quad \underline{e}_{y}=\left[\begin{array}{l}
\frac{\partial \xi}{\partial y} \\
\frac{\partial \eta}{\partial y}
\end{array}\right] .
\end{gathered}
$$

Denoting by $d$ the number of geometrical dimensions of the problem $(d=2$ in the present work) and by $\underline{p}$ the $d \times 1$ position vector in global coordinates, we can formally introduce the $3(d-1) \times d$ matrix operator of partial differentiation $\underline{\underline{\underline{B}}}$ such that

$$
\underline{\varepsilon}(\underline{p})=\underline{\underline{B}} \cdot \underline{u}(\underline{p}),
$$

where $\underline{u}$ and $\underline{\varepsilon}$ are respectively the $d \times 1$ displacement vector and the $3(d-1) \times$ 1 strain vector in Voigt notation. Denoting by $n$ the number of nodes of a generic element $(n=s \times m$ where $s$ represents the number of sides of the element and $m$ the order of the shape functions), we can furthermore introduce the $d \times d \cdot n$ matrix $\underline{\underline{N}}$ of shape functions such that

$$
\underline{u}=\underline{\underline{N}} \cdot \underline{u}_{N}
$$


where $\underline{u}_{N}$ is the $d \cdot n \times 1$ vector of element nodal variables. Having introduced $\underline{\underline{B}}$ and $\underline{\underline{N}}$ in Equations A.4 and A.5 respectively, it is possible to define the $3(d-1) \times d \cdot n$ matrix $\underline{\underline{B}}$ of derivatives (with respect to global coordinates) of shape functions as

$$
\underline{\underline{B}}=\underline{\underline{\widetilde{B}}} \cdot \underline{\underline{N}}
$$

We introduce the linear elastic material behavior in the form of the $3(d-1) \times$ ${ }_{465} 3(d-1)$ rigidity matrix $\underline{\underline{D}}$ such that

$$
\underline{\sigma}=\underline{\underline{D}} \cdot \underline{\varepsilon},
$$

where $\underline{\sigma}$ the $3(d-1) \times 1$ stress vector in Voigt notation. It is finally possible to define the $n \times n$ element stiffness matrix $\underline{\underline{k_{e}}}$ as

$$
\underline{\underline{k_{e}}}=\int_{V_{e}(x, y)}\left(\underline{\underline{B}}^{T} \underline{\underline{D}} \cdot \underline{\underline{B}}\right) d V_{e}(x, \ldots, y)=\int_{V_{e}(\xi, \eta)}\left(\underline{\underline{B}}^{T} \underline{\underline{D}} \cdot \underline{\underline{B}}\right) \sqrt{g} d V_{e}(\xi, \ldots, \eta),
$$

where $g=\operatorname{det}\left(\underline{\underline{J}}^{T} \underline{\underline{J}}\right)$ and $V_{e}$ is the element volume. Given that isoparametric elements are always defined between -1 and 1 in each dimension, Equation A.8 can simplified to

$$
\underline{\underline{k_{e}}}=\int_{-1}^{1} \ldots \int_{-1}^{1}\left(\underline{\underline{B}}^{T} \underline{\underline{D}} \cdot \underline{\underline{B}}\right) \sqrt{g} d \xi, \ldots, d \eta,
$$

which is amenable to numerical integration by means of a Gaussian quadrature of the form

$$
\underline{\underline{k_{e}}} \approx \sum_{i=1}^{N} \cdots \sum_{j=1}^{N} w_{i} \ldots w_{j}\left(\underline{\underline{B}}^{T}\left(\xi_{i}, \ldots, \eta_{j}\right) \cdot \underline{\underline{D}} \cdot \underline{\underline{B}}\left(\xi_{i}, \ldots, \eta_{j}\right) \sqrt{g}\right),
$$

where $\left(\xi_{i}, \ldots, \eta_{j}\right)$ are the coordinates of the $N$ Gaussian quadrature points. The element stiffness matrix as evaluated in Eq. A.10 is in general a full sym- 
metric (in the case of linear elasticity) matrix of the form

$$
k_{e}=\left[\begin{array}{llllllll}
k_{e \mid 11} & k_{e \mid 12} & k_{e \mid 13} & k_{e \mid 14} & k_{e \mid 15} & k_{e \mid 16} & k_{e \mid 17} & k_{e \mid 18} \\
k_{e \mid 12} & k_{e \mid 22} & k_{e \mid 23} & k_{e \mid 24} & k_{e \mid 25} & k_{e \mid 26} & k_{e \mid 27} & k_{e \mid 28} \\
k_{e \mid 13} & k_{e \mid 23} & k_{e \mid 33} & k_{e \mid 34} & k_{e \mid 35} & k_{e \mid 36} & k_{e \mid 37} & k_{e \mid 38} \\
k_{e \mid 14} & k_{e \mid 24} & k_{e \mid 34} & k_{e \mid 44} & k_{e \mid 45} & k_{e \mid 46} & k_{e \mid 47} & k_{e \mid 48} \\
k_{e \mid 15} & k_{e \mid 25} & k_{e \mid 35} & k_{e \mid 45} & k_{e \mid 55} & k_{e \mid 56} & k_{e \mid 57} & k_{e \mid 58} \\
k_{e \mid 16} & k_{e \mid 26} & k_{e \mid 36} & k_{e \mid 46} & k_{e \mid 56} & k_{e \mid 66} & k_{e \mid 67} & k_{e \mid 68} \\
k_{e \mid 17} & k_{e \mid 27} & k_{e \mid 37} & k_{e \mid 47} & k_{e \mid 57} & k_{e \mid 67} & k_{e \mid 77} & k_{e \mid 78} \\
k_{e \mid 18} & k_{e \mid 28} & k_{e \mid 38} & k_{e \mid 48} & k_{e \mid 58} & k_{e \mid 68} & k_{e \mid 78} & k_{e \mid 88}
\end{array}\right] .
$$

Appendix A.2. Calculation of displacements and reaction forces

With reference to Fig. A.10, we define:

$u_{x, M}, u_{x, F}$ the $x$-displacement of the nodes belonging to the free side of the first element belonging to the crack, respectively on the matrix (bulk) and fiber (inclusion) side;

$u_{y, M}, u_{y, F}$ the $y$-displacement of the nodes belonging to the free side of the first element belonging to the crack, respectively on the matrix (bulk) and fiber (inclusion) side;

$u_{r, M}, u_{r, F}$ the $x$-displacement of the nodes belonging to the free side of the first element belonging to the crack, respectively on the matrix (bulk) and fiber (inclusion) side;

$u_{\theta, M}, u_{\theta, F}$ the $y$-displacement of the nodes belonging to the free side of the first element belonging to the crack, respectively on the matrix (bulk) and fiber (inclusion) side;

$F_{x, C T}, F_{y, C T}$ respectively the $x$ - and $y$-component of the reaction force at the crack tip;

$F_{r, C T}, F_{\theta, C T}$ respectively the $r$ - and $\theta$-component of the reaction force at the crack tip. 
The $x-y$ reference frame is the global reference frame, while the $r-\theta$ reference frame is such that the $\theta$ direction coincides with the crack propagation direction at the crack tip and $r$ the in-plane normal to the propagation direction. For an arc-crack as the present one, the $r$-direction coincides with the radial direction of the inclusion.

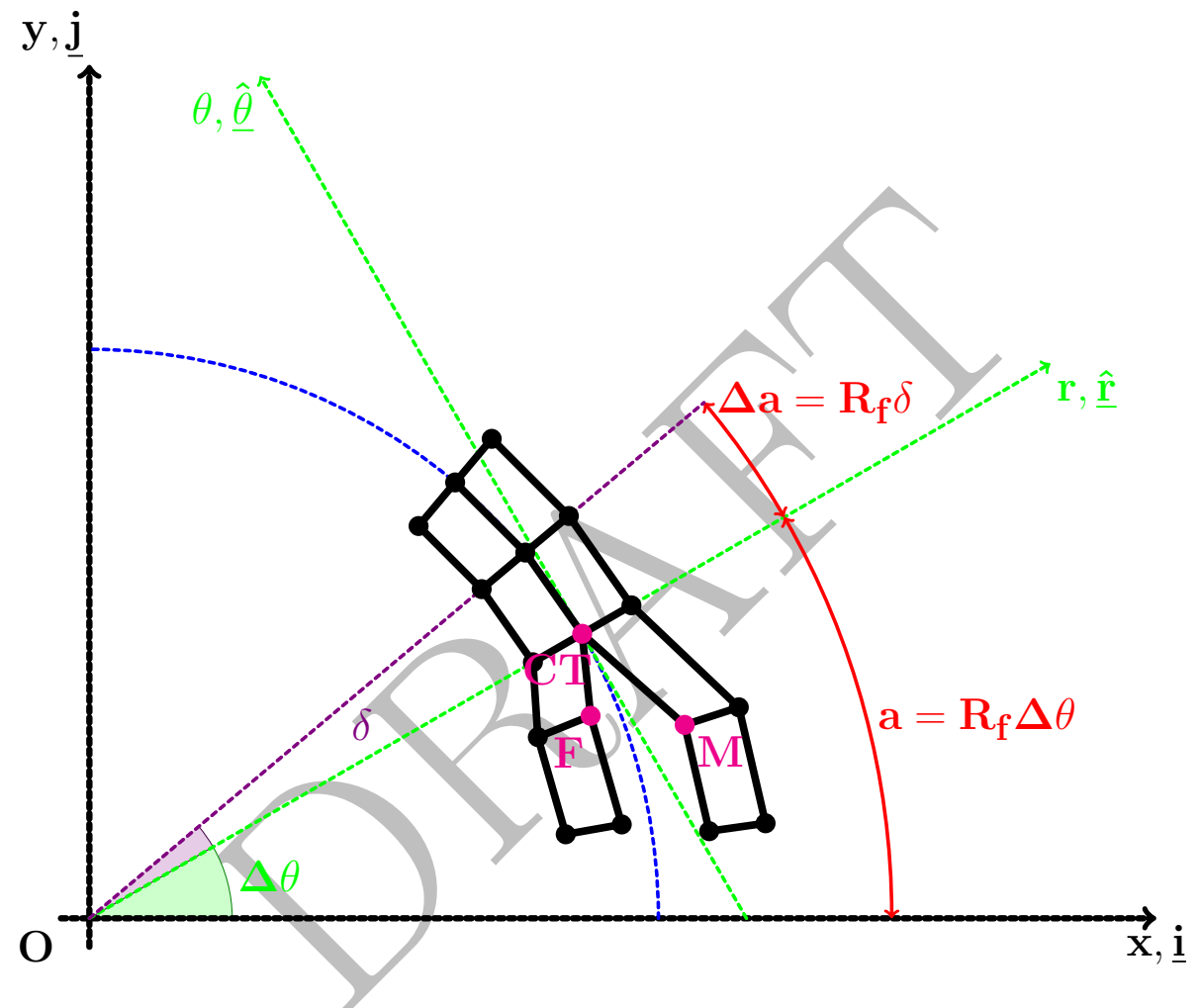

Figure A.10: Schematic representation of the discretized crack tip geometry for $1^{\text {st }}$ order quadrilateral elements.

The crack opening displacement $u_{r}$ and the crack shear displacement $u_{\theta}$ at 500 the crack tip can thus be written as

$$
u_{r}=\cos (\Delta \theta) u_{x}+\sin (\Delta \theta) u_{y} \quad u_{\theta}=-\sin (\Delta \theta) u_{x}+\cos (\Delta \theta) u_{y}
$$


where $u_{x}$ and $u_{y}$ are defined as

$$
u_{x}=u_{x, M}-u_{x, F} \quad u_{y}=u_{y, M}-u_{y, F}
$$

and $2 \Delta \theta$ is total angular size of the debond. The corresponding forces at the crack tip are

$F_{r}=\cos (\Delta \theta) F_{x, C T}+\sin (\Delta \theta) F_{y, C T} \quad F_{\theta}=-\sin (\Delta \theta) F_{x, C T}+\cos (\Delta \theta) F_{y, C T}$.

At the crack tip, the FE mesh possesses two coincident points, labeled $F C T$ and $M C T$. Continuity of the displacements at the crack tip must be ensured. Furthermore, in order to measure the force at the crack tip, a fully-constraint dummy node needs to be created and formally linked to the two nodes at the crack tip by the conditions

which can be simplified to

$$
\left\{\begin{array}{l}
u_{x, F C T}-u_{x, M C T}-u_{x, D U M M Y}=0 \\
u_{y, F C T}-u_{y, M C T}-u_{y, D U M M Y}=0 \\
u_{x, D U M M Y}=0 \\
u_{y, D U M M Y}=0
\end{array}\right.
$$

$$
\left\{\begin{array}{l}
u_{x, F C T}=u_{x, M C T} \\
u_{y, F C T}=u_{y, M C T} \\
R_{x, D U M M Y}=R_{x, F C T}=-R_{x, M C T}=F_{x, C T} \\
R_{y, D U M M Y}=R_{y, F C T}=-R_{y, M C T}=F_{y, C T}
\end{array} .\right.
$$

${ }_{510}$ Making use of Eq. A.11, four equations can be written in the four displace- 
ment $u_{x, F C T}, u_{x, M C T}, u_{y, F C T}$ and $u_{y, M C T}$ :

$$
\begin{aligned}
& \left(k_{e, M \mid 11}+k_{e, M \mid 33}\right) u_{x, M C T}+\left(k_{e, M \mid 12}+k_{e, M \mid 34}\right) u_{y, M C T}+ \\
& +k_{e, M \mid 13} u_{x, M}+k_{e, M \mid 14} u_{y, M}+\left(k_{M \mid 17}+k_{M \mid 35}\right) u_{N, M C \mid 7}+\left(k_{M \mid 18}+k_{M \mid 36}\right) u_{N, M C \mid 8}+ \\
& +\sum_{i=5}^{6} k_{M \mid 1 i} u_{N, M C \mid i}+\sum_{i=7}^{8} k_{M \mid 3 i} u_{N, M B \mid i}+k_{M \mid 31} u_{x, N C O I}+k_{M \mid 32} u_{y, N C O I}=0 \\
& \left(k_{e, M \mid 21}+k_{e, M \mid 43}\right) u_{x, M C T}+\left(k_{e, M \mid 22}+k_{e, M \mid 44}\right) u_{y, M C T}+ \\
& +k_{e, M \mid 23} u_{x, M}+k_{e, M \mid 24} u_{y, M}+\left(k_{M \mid 27}+k_{M \mid 45}\right) u_{N, M C \mid 7}+\left(k_{M \mid 28}+k_{M \mid 46}\right) u_{N, M C \mid 8}+ \\
& +\sum_{i=5}^{6} k_{M \mid 2 i} u_{N, M C \mid i}+\sum_{i=7}^{8} k_{M \mid 4 i} u_{N, M B \mid i}+k_{M \mid 41} u_{x, N C O I}+k_{M \mid 42} u_{y, N C O I}=0 \\
& \left(k_{e, F \mid 77}+k_{e, F \mid 55}\right) u_{x, F C T}+\left(k_{e, F \mid 78}+k_{e, F \mid 56}\right) u_{y, F C T}+ \\
& +k_{e, F \mid 75} u_{x, F}+k_{e, F \mid 76} u_{y, F}+\left(k_{F \mid 71}+k_{F \mid 53}\right) u_{N, F C \mid 1}+\left(k_{F \mid 72}+k_{F \mid 54}\right) u_{N, F C \mid 2}+ \\
& +\sum_{i=2}^{3} k_{F \mid 7 i} u_{N, F C \mid i}+\sum_{i=1}^{2} k_{F \mid 5 i} u_{N, F B \mid i}+k_{F \mid 57} u_{x, N C O I}+k_{F \mid 58} u_{y, N C O I}=0 \\
& \left(k_{e, F \mid 87}+k_{e, F \mid 65}\right) u_{x, F C T}+\left(k_{e, F \mid 88}+k_{e, F \mid 66}\right) u_{y, F C T}+ \\
& +k_{e, F \mid 85} u_{x, F}+k_{e, F \mid 86} u_{y, F}+\left(k_{F \mid 81}+k_{F \mid 63}\right) u_{N, F C \mid 1}+\left(k_{F \mid 82}+k_{F \mid 64}\right) u_{N, F C \mid 2}+ \\
& +\sum_{i=2}^{3} k_{F \mid 8 i} u_{N, F C \mid i}+\sum_{i=1}^{2} k_{F \mid 6 i} u_{N, F B \mid i}+k_{F \mid 67} u_{x, N C O I}+k_{F \mid 68} u_{y, N C O I}=0
\end{aligned}
$$

Solving for $u_{y, F C T}$ and $u_{y, M C T}$ the third and fourth relations in Eq. A.17 
and substituting in the first two expressions of Eq. A.17, we get

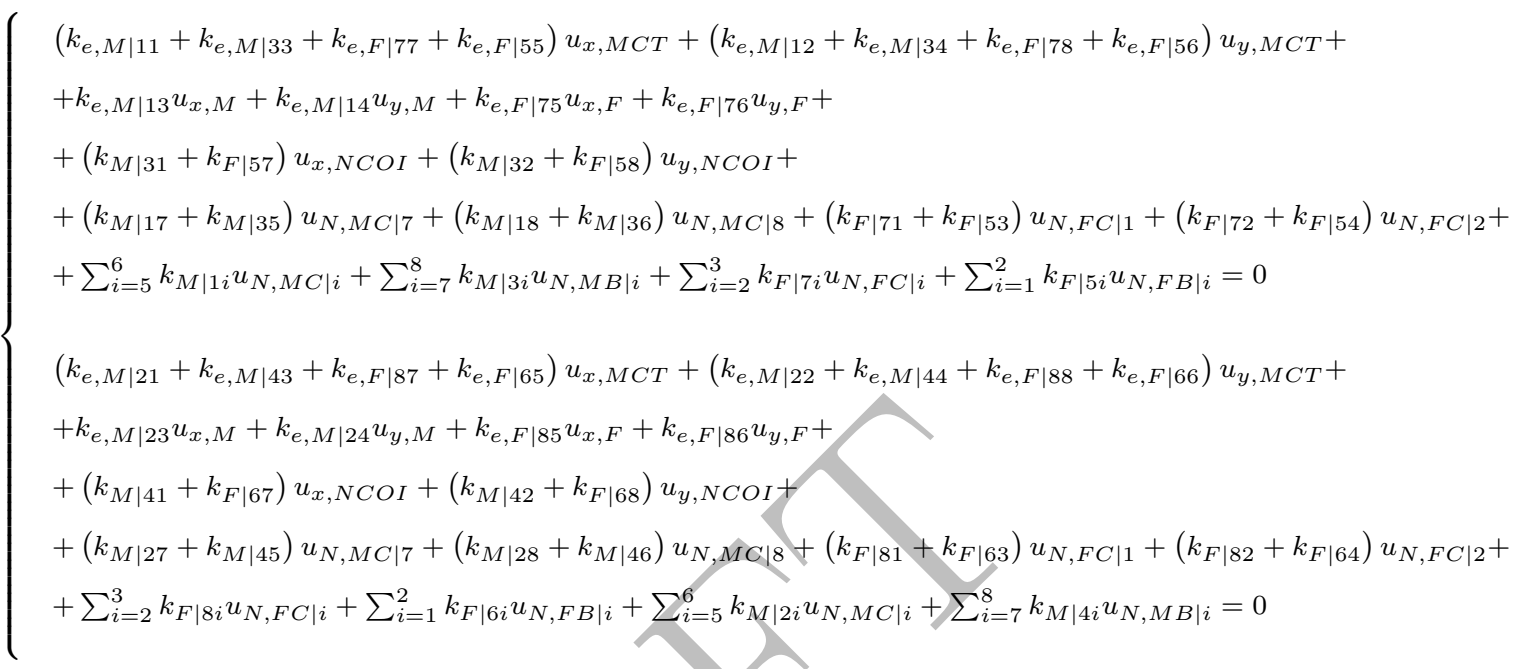

Solving the system of two equations and observing that $u_{x, F}, u_{y, F} \sim 0$ for a 515 stiffer inclusion as a fiber in a polymeric composite, we can express $u_{x, M C T}$ as

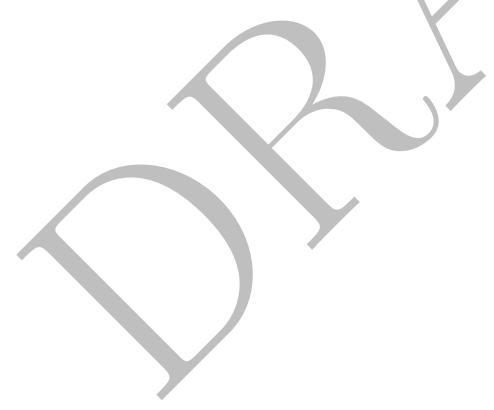


a function of $u_{x}$ and $u_{y}$ (see Eq. A.13) as

$$
\begin{aligned}
& {\left[\left(k_{e, M \mid 21}+k_{e, M \mid 43}+k_{e, F \mid 87}+k_{e, F \mid 65}\right)+\frac{k_{e, M \mid 11}+k_{e, M \mid 33}+k_{e, F \mid 77}+k_{e, F \mid 55}}{k_{e, M \mid 12}+k_{e, M \mid 34}+k_{e, F \mid 78}+k_{e, F \mid 56}}\left(k_{e, M \mid 22}+k_{e, M \mid 44}+k_{e, F \mid 88}+k_{e, F \mid 66}\right)\right] u_{x, M C T}+} \\
& +\left(k_{e, M \mid 23}-\frac{k_{e, M \mid 22}+k_{e, M \mid 44}+k_{e, F \mid 88}+k_{e, F \mid 66}}{k_{e, M \mid 12}+k_{e, M \mid 34}+k_{e, F \mid 78}+k_{e, F \mid 56}} k_{e, M \mid 13}\right) u_{x}+ \\
& +\left(k_{e, M \mid 24}-\frac{k_{e, M \mid 22}+k_{e, M \mid 44}+k_{e, F \mid 88}+k_{e, F \mid 66}}{k_{e, M \mid 12}+k_{e, M \mid 34}+k_{e, F \mid 78}+k_{e, F \mid 56}} k_{e, M \mid 14}\right) u_{y}+ \\
& +\left(k_{e, M \mid 23}+k_{e, F \mid 85}-\frac{k_{e, M \mid 22}+k_{e, M \mid 44}+k_{e, F \mid 88}+k_{e, F \mid 66}}{k_{e, M \mid 12}+k_{e, M \mid 34}+k_{e, F \mid 78}+k_{e, F \mid 56}}\left(k_{e, M \mid 13}+k_{e, M \mid 75}\right)\right) u_{\text {. }} \approx+ \\
& +\left(k_{e, M \mid 24}+k_{e, F \mid 86}-\frac{k_{e, M \mid 22}+k_{e, M \mid 44}+k_{e, F \mid 88}+k_{e, F \mid 66}}{k_{e, M \mid 12}+k_{e, M \mid 34}+k_{e, F \mid 78}+k_{e, F \mid 56}}\left(k_{e, M \mid 14}+k_{e, M \mid 76}\right)\right) u_{y, F} \approx+ \\
& +\left[\left(k_{M \mid 41}+k_{F \mid 67}\right)-\frac{k_{e, M \mid 22}+k_{e, M \mid 44}+k_{e, F \mid 88}+k_{e, F \mid 66}}{k_{e, M \mid 12}+k_{e, M \mid 34}+k_{e, F \mid 78}+k_{e, F \mid 56}}\left(k_{M \mid 31}+k_{F \mid 57}\right)\right] u_{x, N C O I}+ \\
& +\left[\left(k_{M \mid 42}+k_{F \mid 68}\right)-\frac{k_{e, M \mid 22}+k_{e, M \mid 44}+k_{e, F \mid 88}+k_{e, F \mid 66}}{k_{e, M \mid 12}+k_{e, M \mid 34}+k_{e, F \mid 78}+k_{e, F \mid 56}}\left(k_{M \mid 32}+k_{F \mid 58}\right)\right] u_{y, N C O I}+ \\
& +\left(k_{M \mid 27}+k_{M \mid 45}\right) u_{N, M C \mid 7}+\left(k_{M \mid 28}+k_{M \mid 46}\right) u_{N, M C \mid 8}+\left(k_{F \mid 81}+k_{F \mid 63}\right) u_{N, F C \mid 1}+\left(k_{F \mid 82}+k_{F \mid 64}\right) u_{N, F C \mid 2}+ \\
& -\frac{k_{e, M \mid 22}+k_{e, M \mid 44}+k_{e, F \mid 88}+k_{e, F \mid 66}\left[\left(k_{M \mid 17}+k_{M \mid 35}\right) u_{N, M C \mid 7}+\left(k_{M \mid 18}+k_{M \mid 36}\right) u_{N, M C \mid 8}\right]+}{k_{e, M \mid 12}+k_{e, M \mid 34}+k_{e, F \mid 78}+k_{e, F \mid 56}} \\
& -\frac{k_{e, M \mid 22}+k_{e, M \mid 44}+k_{e, F \mid 88}+k_{e, F \mid 66}\left[\left(k_{F \mid 71}+k_{F \mid 53}\right) u_{N, F C \mid 1}+\left(k_{F \mid 72}+k_{F \mid 54}\right) u_{N, F C \mid 2}\right]}{k_{e, M \mid 12}+k_{e, M \mid 34}+k_{e, F \mid 78}+k_{e, F \mid 56}} \\
& +\sum_{i=2}^{3} k_{F \mid 8 i} u_{N, F C \mid i}+\sum_{i=1}^{2} k_{F \mid 6 i} u_{N, F B \mid i}+\sum_{i=5}^{6} k_{M \mid 2 i} u_{N, M C \mid i}+\sum_{i=7}^{8} k_{M \mid 4 i} u_{N, M B \mid i}+ \\
& -\frac{\sum_{i=5}^{6} k_{M \mid 1 i} u_{N, M C \mid i}+\sum_{i=7}^{8} k_{M \mid 3 i} u_{N, M B \mid i}+\sum_{i=2}^{3} k_{F \mid 7 i} u_{N, F C \mid i}+\sum_{i=1}^{2} k_{F \mid 5 i} u_{N, F B \mid i}}{k_{e, M} \mid 12+k_{e, M \mid 34}+k_{e, F \mid 78}+k_{e, F \mid 56}}=0
\end{aligned}
$$

while the reaction forces at the crack tip can be expressed as

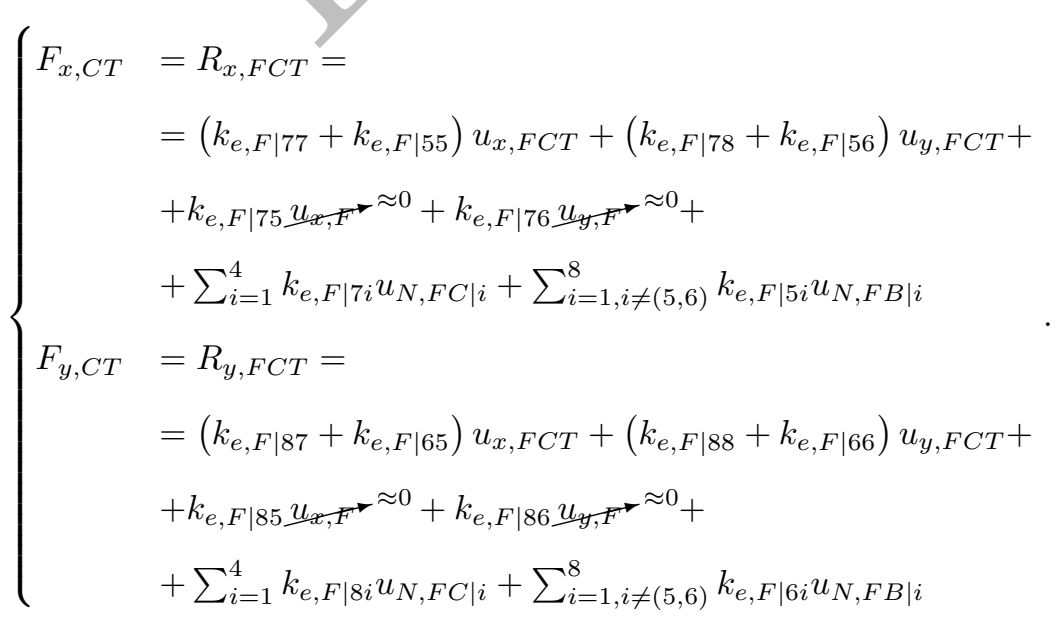


Substituting Eq. A.17 in Eq. A.18, Eq. A.19 and Eq. A.20 and solving, we obtain an expression of the form

$$
\left\{\begin{array}{rl}
F_{x, C T} & =K_{x x} u_{x}+K_{x y} u_{y}+ \\
& +\sum_{i=1}^{4} K_{F C, x \mid i} u_{N, F C \mid i}+\sum_{i=1, i \neq(3,4,5,6)}^{8} K_{F B, x \mid i} u_{N, F B \mid i}+ \\
& +\sum_{i=5}^{8} K_{F C, x \mid i} u_{N, M C \mid i}+\sum_{i=7}^{8} K_{M B, x \mid i} u_{N, F B \mid i} \\
F_{y, C T} & =K_{y x} u_{x}+K_{y y} u_{y}+ \\
& +\sum_{i=1}^{4} K_{F C, y \mid i} u_{N, F C \mid i}+\sum_{i=1, i \neq(3,4,5,6)}^{8} K_{F B, y \mid i} u_{N, F B \mid i}+ \\
& +\sum_{i=5}^{8} K_{F C, y \mid i} u_{N, M C \mid i}+\sum_{i=7}^{8} K_{M B, y \mid i} u_{N, F B \mid i}
\end{array},\right.
$$

which can be reformulated synthetically as

$$
\left\{\begin{array}{l}
F_{x, C T}=K_{x x} u_{x}+K_{x y} u_{y}+\widetilde{F}_{x} \\
F_{y, C T}=K_{y x} u_{x}+K_{y y} u_{y}+\widetilde{F}_{y}
\end{array}\right.
$$

where $\widetilde{F}_{x}$ and $\widetilde{F}_{y}$ represent the influence of the FE solution through the nodes of the elements sharing the crack tip that do not belong to any of the phase interfaces, i.e. the nodes of the elements sharing the crack tip that belong to the bulk of each phase. 


\section{Appendix B. Expression of $\underline{\underline{T}}_{p q}$ for quadrilateral elements with or without singularity}

The expression of $\underline{\underline{T}}_{p q}$ for quadrilateral elements with or without singularity is

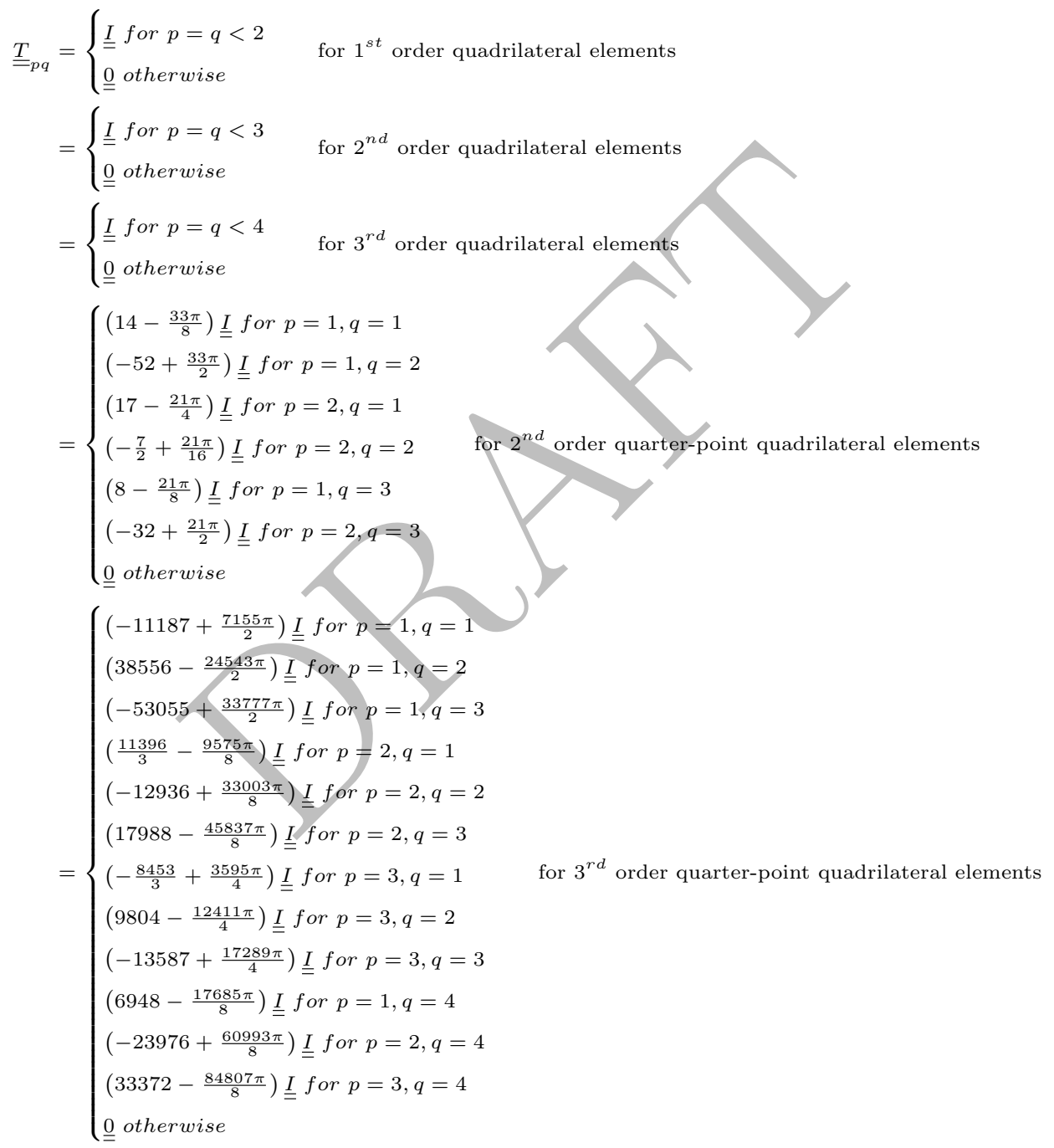

where $\underline{\underline{I}}$ is the identity matrix. 